\title{
Ship hydrodynamic optimization by local hybridization of deterministic derivative-free global algorithms
}

\author{
Andrea Serani ${ }^{\mathrm{a}, \mathrm{b}}$, Giovanni Fasano ${ }^{\mathrm{c}}$, Giampaolo Liuzzi ${ }^{\mathrm{d}}$, Stefano Lucidi ${ }^{\mathrm{e}}$, \\ Umberto Iemma $^{\mathrm{b}}$, Emilio F. Campana ${ }^{\mathrm{a}}$, Frederick Stern ${ }^{\mathrm{f}}$, Matteo Diez ${ }^{\mathrm{a}, *}$ \\ a CNR-INSEAN, Natl. Research Council - Marine Technology Research Inst., Rome, Italy \\ b Department of Engineering, Roma Tre University, Rome, Italy \\ ' Department of Management, Ca' Foscari University of Venice, Venice, Italy \\ d CNR-IASI, Natl. Research Council - Inst. for Systems Analysis and Computer Science, Rome, Italy \\ e Department of Computer, Control, and Management Engineering “A. Ruberti”, Sapienza University of Rome, Rome, Italy \\ ${ }^{\mathrm{f}}$ IIHR - Hydroscience and Engineering, The University of Iowa, Iowa City, IA, USA
}

\section{A R T I C L E I N F O}

\section{Article history:}

Received 13 November 2015

Received in revised form 5 April 2016

Accepted 17 April 2016

\section{Keywords:}

Simulation-based design

Ship hydrodynamics

Hull-form optimization

Particle swarm optimization

DIRECT

Line search methods

Hybrid global/local algorithms

DTMB 5415

\begin{abstract}
A B S T R A C T
Simulation-based design optimization methods integrate computer simulations, design modification tools, and optimization algorithms. In hydrodynamic applications, often objective functions are computationally expensive and noisy, their derivatives are not directly provided, and the existence of local minima cannot be excluded a priori, which motivates the use of deterministic derivative-free global optimization algorithms. The enhancement of two algorithms of this type, DIRECT (DIviding RECTangles) and DPSO (Deterministic Particle Swarm Optimization), is presented based on global/local hybridization with derivative-free line search methods. The hull-form optimization of the DTMB 5415 model is solved for the reduction of the calm-water resistance at $\mathrm{Fr}=0.25$, using potential flow and RANS solvers. Six and eleven design variables are used respectively, modifying both the hull and the sonar dome. Hybrid algorithms show a faster convergence towards the global minimum than the original global methods and are a viable option for ship hydrodynamic optimization. A significant resistance reduction is achieved both by potential flow and RANS-based optimizations, showing the effectiveness of the optimization procedure.
\end{abstract}

(c) 2016 Elsevier Ltd. All rights reserved.

\section{Introduction}

In the last decades, the design process of ship hulls, propellers, and complex ocean engineering systems has experienced a significant paradigm shift, due to the availability of high-performance computing systems (hardware) and accurate physics-based solvers (software). The traditional and expensive build and test paradigm has been replaced by the more advanced and flexible simulationbased design (SBD) approach, which integrates computer simulations, design modification methods, and possibly optimization algorithms [1-4]. In SBD, an inverse problem is solved, providing as result the design that maximizes the performance and/or minimizes the cost (objective function), given a set of design specifications (constraint functions). In SBD optimization for ship and ocean engineering applications, objective and constraint functions are usually provided by systems of partial differential equations, often

\footnotetext{
* Corresponding author.

E-mail address: matteo.diez@cnr.it (M. Diez).
}

solved by black-box tools. In this context, the functions are likely affected by residuals, therefore noisy, and their derivatives are not directly provided. Often, the existence in the design space of local minima cannot be excluded a priori. For these reasons, derivativefree global optimization algorithms have been developed, providing a global approximate solution to the design problem [5].

Derivative-free global algorithms have emerged as powerful methods, in order to tackle complex optimization problems in many engineering fields, including ship and ocean engineering. The robustness and versatility of these methods have allowed for their successful application not only to design optimization, but also to identification and prediction of complex hydrodynamic systems. Recently, a genetic algorithm has been applied to the shape optimization of axisymmetric cavitators in supercavitating flows [6]. The artificial bee colony has been applied to identify berm geometries using a set of laboratory tests combined with teaching-learning-based optimization [7]. Five derivative-free global algorithms of the evolutionary type (genetic algorithm, memetic algorithm, particle swarm optimization, ant colony optimization, and shuffled frog leaping algorithm) have been assessed 
and compared in the 3D path optimization of underwater vehicles [8]. Derivative-free global methods (such as genetic algorithms and particle swarm optimization) have been applied to the hull form optimization of a destroyer, within the research activities of the NATO Science and Technology Organization, Applied Vehicle Technology (AVT) task groups 204 "Assess the Ability to Optimize Hull Forms of Sea Vehicles for Best Performance in a Sea Environment" and 252 "Stochastic Design Optimization for Naval and Aero Military Vehicles." When global techniques are used with CPU-time expensive solvers (for hydrodynamics, structures, etc.), the optimization process is computationally expensive and its effectiveness and efficiency remain an algorithmic and technological challenge. Although complex SBD applications are often solved by metamodels $[9,10]$, their development and assessment require benchmark solutions, with simulations directly connected to the optimization algorithm. These solutions are achieved only if affordable and effective optimization procedures are available.

Although global optimization approaches are a good compromise between exploration and exploitation of the research space, they could still get trapped in local minima and the convergence to a global minimum cannot be proven. If the research region to explore is known a priori, local optimization approaches can give an accurate approximation of the local minimum. Nevertheless, their convergence may be computationally expensive, and the information is usually not available a priori. For these reasons, the hybridization of global optimization algorithms with local search methods is an interesting research field, especially if CPU-time expensive black-box functions are involved, where the qualities of both methods can be efficiently and robustly coupled.

Finally, it is worth noting that a large variety of derivative-free global and local methods available in the literature are probabilistic. These methods make use of random coefficients and have been developed to the aim of sustaining the variety of the search for an optimum. This property implies that statistically significant results can be obtained only through extensive numerical campaigns. Such an approach can be too expensive (often almost unaffordable) in SBD optimization for industrial applications, when CPU-time expensive computer simulations are used directly as analysis tools. For this reason, deterministic approaches have been successfully developed and applied to SBD optimization, including hydrodynamic problems [5,11].

The objective of the present work is the local hybridization of two deterministic derivative-free global optimization algorithms, with derivative-free local searches. These methods are presented as a viable and effective option for ship hydrodynamic optimization. Their performance is assessed and compared with that of the original algorithms.

Specifically, four derivative-free global and hybrid global/local optimization algorithms are presented and applied. Two algorithms are well-known global optimization approaches, specifically (a) the DIRECT (DIviding RECTangles) algorithm [12], and (b) a deterministic version of the particle swarm optimization method (DPSO, [13]). The other two algorithms are hybrid global/local techniques integrated in (a) and (b), respectively, enhancing the global methods with proved stationarity of the final solution. A hybrid DIRECT method coupled with line search-based derivative-free optimization, namely DIRMIN-2 [14], and a hybrid DPSO coupled with line search-based derivative-free optimization, namely LS-DF_PSO [15] are presented and applied.

The SBD application presented is the hydrodynamic hull-form optimization of a USS Arleigh Burke-class destroyer, namely the DTMB 5415 model, an early and open to public version of the DDG-51. The DTMB 5415 model has been widely investigated through towing tank experiments $[16,17]$ and SBD studies, including hull-form optimization [18]. Recently, the DTMB 5415 model has been selected as the test case for the SBD activities within the NATO AVT-204, aimed at a multi-objective design optimization for multi-speed reduced resistance and improved seakeeping performance [19], and AVT-252 for stochastic design optimization. Herein, a deterministic single-speed single-objective example is shown, aimed at the reduction of the total resistance in calm water at $18 \mathrm{kn}$, corresponding to Froude number ( $\mathrm{Fr}$ ) equal to 0.25 . The design constraints include fixed displacement and length between perpendicular, along with a $\pm 5 \%$ maximum variation of beam and draft. The ship is free to sink and trim. An expansion of orthogonal basis functions is used for the modification of the hull form and the sonar dome. Two problems are solved and presented. The first (I) is based on potential flow computations. The solver used is a linear potential flow code (WARP) [20], allowing for the evaluation of the wave resistance by transversal wave cut $[21,22]$. The resistance due to friction is estimated by a local approximation based on flat-plate theory [23]. The number of basis functions and design variables is equal to six. This first problem was already addressed in the early work [27], of which the present paper can be considered the natural evolution. The second problem (II) is solved using a RANS solver (CFDShip-Iowa v4.5) [24] with a first order polyharmonic spline metamodel [9]. The basis functions for the shape modification are eleven and are the result of a dimensionality reduction analysis, based on the Karhunen-Loève expansion (KLE) [25,26].

\section{Optimization problem and algorithms}

Consider the following objective function:

$f(\boldsymbol{\alpha}): \mathbb{R}^{N} \longrightarrow \mathbb{R}$

and the global optimization problem

$\min _{\boldsymbol{\alpha} \in \mathcal{L}} f(\boldsymbol{\alpha}), \quad \mathcal{L} \subset \mathbb{R}^{N}$

where $\boldsymbol{\alpha}=\left\{\alpha_{j}\right\}$ is the design variable vector and $\mathcal{L}$ is a closed and bounded subset of $\mathbb{R}^{N}$, identified here by the lower $\left(\ell_{j}\right)$ and upper $\left(u_{j}\right)$ bounds of each design variable $\alpha_{j}$. The global minimization of the objective function $f(\boldsymbol{\alpha})$ requires to find a vector $\mathbf{a} \in \mathcal{L}$ so that:

$\forall \mathbf{b} \in \mathcal{L}: f(\mathbf{a}) \leq f(\mathbf{b})$

Then, $\boldsymbol{\alpha}=\mathbf{a}$ is a global minimum for the function $f(\boldsymbol{\alpha})$ over $\mathcal{L}$. The exact identification of a global minimum might be very difficult, representing a theoretical, methodological, and technological challenge. Therefore, approximate solutions provided by heuristic procedures are often considered acceptable for practical purposes. The deterministic derivative-free global algorithms (DIRECT and DPSO) and their global/local hybridizations (DIRMIN-2 and LSDF_PSO) are presented in the following, for the solution of Eq. (2).

\subsection{The DIRECT algorithm}

DIRECT is a sampling deterministic global derivative-free optimization algorithm and a modification of the Lipschitzian optimization method [12]. It starts the optimization by transforming the search domain $\mathcal{L}$ of the problem into the unit hyper-cube $\mathcal{D}$. At the first step of DIRECT, $f(\boldsymbol{\alpha})$ is evaluated at the center $(c)$ of $\mathcal{D}$; the hyper-cube is then partitioned into a set of smaller hyperrectangles and $f(\boldsymbol{\alpha})$ is evaluated at their centers. Let the partition of $\mathcal{D}$ at iteration $k$ be defined as

$$
\begin{aligned}
& \mathcal{H}_{k}=\left\{\mathcal{D}_{i}: i \in \mathcal{I}_{k}\right\}, \quad \text { with } \\
& \mathcal{D}_{i}=\left\{\boldsymbol{\alpha} \in \mathbb{R}^{N}: \ell_{j}^{(i)} \leq \alpha_{j} \leq u_{j}^{(i)}, \quad j=1, \ldots, N, \quad \forall i \in \mathcal{I}_{k}\right\}
\end{aligned}
$$

where $N$ is the number of design variables, $\ell_{j}^{(i)}$ and $u_{j}^{(i)} \in[0,1]$, with $i \in \mathcal{I}_{k}$, are the lower and upper bounds defining the hyperrectangle $\mathcal{D}_{i}$, and $\mathcal{I}_{k}$ is the set of indices identifying the subsets 
defining the current partition. At a generic $k$ th iteration of the algorithm, starting from the current partition $\mathcal{H}_{k}$ of $\mathcal{D}$, a new partition, $\mathcal{H}_{k+1}$, is built by subdividing a set of promising hyper-rectangles of the previous one. The identification of "potentially optimal" hyperrectangles is based on some measure of the hyper-rectangle itself and on the value of $f(\boldsymbol{\alpha})$ at its center $c^{i}$. The refinement of the partition continues until a prescribed number of function evaluations have been performed, or another stopping criterion is satisfied. The minimum of $f(\boldsymbol{\alpha})$ over all the centers of the final partition, and the corresponding center, provide an approximate solution to the problem. It may be noted that the box constraints are automatically satisfied.

\subsection{Local hybridization of the DIRECT algorithm: DIRMIN-2}

DIRMIN-2 is a global/local hybridization of the DIRECT algorithm and a variant of DIRMIN [28,14]. Differently from DIRMIN, that performs as many local searches as the number of identified potentially optimal hyper-rectangles, DIRMIN-2 performs a single derivativefree local minimization per iteration, starting from the best point produced by dividing the potentially-optimized hyper-rectangles. DIRMIN-2's local minimization is used when the number of function evaluations reaches the activation trigger $\gamma \in(0,1)$, a ratio of the maximum number of function evaluations $\left(N_{f_{\max }}\right)$. The local minimization proceeds until either the number of function evaluations exceeds $N_{f_{\max }}$ or the step size $\Delta$ falls below a given tolerance $\beta$. The local search is not allowed to violate the box constraints. The performance of the algorithm varying the tolerance $\beta$ and the activation trigger $\gamma$ has been studied in [14], where DIRMIN-2 is applied to a ship optimization problem. Herein the following set up are used: $\gamma=0.25, \beta=10^{-3}$ for problem I, and $\gamma=0, \beta=10^{-2}$ for problem II.

\subsection{The DPSO algorithm}

Particle Swarm Optimization (PSO) was originally introduced in [29], based on the social-behavior metaphor of a flock of birds or a swarm of bees searching for food. PSO belongs to the class of heuristic algorithms for single-objective evolutionary derivativefree global optimization. In order to make PSO more efficient for use within SBD, a deterministic version of the algorithm (DPSO) was formulated in [5] as follows

$\left\{\begin{array}{l}\mathbf{v}_{i}^{k+1}=\chi\left[\mathbf{v}_{i}^{k}+c_{1}\left(\boldsymbol{\alpha}_{i, p b}-\boldsymbol{\alpha}_{i}^{k}\right)+c_{2}\left(\boldsymbol{\alpha}_{g b}-\boldsymbol{\alpha}_{i}^{k}\right)\right] \\ \boldsymbol{\alpha}_{i}^{k+1}=\boldsymbol{\alpha}_{i}^{k}+\mathbf{v}_{i}^{k+1}\end{array}\right.$

The above equations update velocity and position of the $i$ th particle at the $k$ th iteration, where $\chi$ is the constriction factor; $c_{1}$ and $c_{2}$ are the social and cognitive learning rate; $\boldsymbol{\alpha}_{i, p b}$ is the personal best position ever found by the $i$ th particle and $\boldsymbol{\alpha}_{g b}$ is the global best position ever found by all particles. A systematic study on the performance and the use of DPSO, defining a guideline, successfully applied on a ship design optimization problem has been made in [13]. Herein the setup suggested in $[13,30]$ are used: (Problem I) number of particles $N_{p}=4 N$, particles initialization by Hammersly Sequence Sampling (HSS, [31]) distribution on domain and bounds with non-null velocity, set of coefficient $\chi=0.721, c_{1}=c_{2}=1.655$ [32], semi-elastic wall-type approach for box constraints, and (Problem II) $N_{p}=16 N$, particles initialization by HSS distribution on domain with non-null velocity, set of coefficient $\chi=0.600, c_{1}=c_{2}=1.700$ [33], semi-elastic wall-type approach for box constraints. Specifically, in the case a particle violates one of the box constraints, then the $i$ th particle is placed on the active

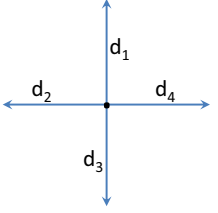

Fig. 1. Example of PSS in $\mathbb{R}^{2}$.

boundary while the associated $j$ th velocity component is redefined as

$v_{i}^{j}=\frac{-v_{i}^{j}}{\left[\chi\left(c_{1}+c_{2}\right)\right]}$

\subsection{Local hybridization of the DPSO algorithm: LS-DF_PSO}

Global convergence properties of a modified PSO scheme may be obtained by properly combining PSO with a line search-based derivative-free method, so that convergence to stationary points can be forced at a reasonable cost. Ref. [15] provides a robust method to force the convergence of a subsequence of points toward a stationary point, which satisfies first order optimality conditions for the objective function. The method, namely LS-DF_PSO, starts by coupling the DPSO scheme with a line search-based method. Specifically, a Positively Spanning Set (PSS) is used, where the set of search directions $\left(D_{\oplus}\right)$ is defined by the unit vectors $\pm e_{i}, i=1, \ldots$, $N$, as shown in the following equation (i.e., $N=2$ ) and in Fig. 1 .

$D_{\oplus}=\left\{\left(\begin{array}{l}0 \\ 1\end{array}\right),\left(\begin{array}{c}-1 \\ 0\end{array}\right),\left(\begin{array}{c}0 \\ -1\end{array}\right),\left(\begin{array}{l}1 \\ 0\end{array}\right)\right\}$

After each DPSO iteration, the local search from the best particle is performed if the swarm has not find a new global minimum. The initial step size $\left(\zeta^{k}\right)$ for the local search is set equal to 0.25 times the variable domain range, and it is reduced by $\vartheta=0.5$ at each local search iteration. Local searches continue in each direction until the step size is greater than $\mu=10^{-3}$. If the local search stops without providing a new global minimum, the actual global minimum is declared as a stationary point. The line search method is not allowed to violate the box constraints.

\section{Hull form optimization problems}

Fig. 2 shows the geometry of the CNR-INSEAN 2340 model, a geosym replica of the DTMB 5415 model used for towing tank experiments, as seen in [16]. The main particulars of the full/model scale and tests conditions are summarized in Table 1 . Since no rudder is considered here, the length between perpendiculars $(L B P)$ is calculated from the fore perpendicular to the transom bottom edge.

The objective function is the total resistance $\left(R_{T}\right)$ (consequently the total resistance coefficient, $C_{T}$ ) in calm water at $\mathrm{Fr}=0.25$. The full scale is considered for Problem I, whereas the model scale is considered for Problem II.

Shape modifications $\boldsymbol{\delta}_{S}$ are produced directly on the Cartesian coordinates $\mathbf{x}_{s}$ of the computational body surface grid, as per

$\mathbf{x}_{S}(\boldsymbol{\alpha})=\mathbf{x}_{s, 0}+\boldsymbol{\delta}_{s}(\boldsymbol{\alpha})$

where $\boldsymbol{\alpha}$ is the design variable vector and $\mathbf{x}_{s, 0}$ represents the original body surface grid. An orthogonal expansion of the shape

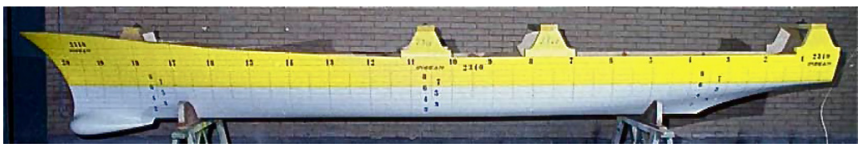

Fig. 2. A $5.720 \mathrm{~m}$ length model of the DTMB 5415 (CNR-INSEAN model 2340). 
Table 1

DTMB 5415 main particulars and test conditions.

\begin{tabular}{|c|c|c|c|c|}
\hline Description & Symbol & Unit & Full scale & $\begin{array}{l}\text { Model } \\
\text { scale }\end{array}$ \\
\hline Displacement & $\nabla$ & (tonnes) & 8636 & 0.549 \\
\hline $\begin{array}{l}\text { Length between } \\
\text { perpendiculars }\end{array}$ & $L B P$ & $(\mathrm{~m})$ & 142.0 & 5.720 \\
\hline Beam & $B$ & $(\mathrm{~m})$ & 18.90 & 0.760 \\
\hline Draft & $T$ & $(\mathrm{~m})$ & 6.160 & 0.248 \\
\hline $\begin{array}{l}\text { Longitudinal center of } \\
\text { gravity }\end{array}$ & $L C G$ & $(\mathrm{~m})$ & 71.60 & 2.884 \\
\hline $\begin{array}{l}\text { Vertical center of } \\
\text { gravity }\end{array}$ & VCG & $(\mathrm{m})$ & 1.390 & 0.056 \\
\hline Froude number & $\mathrm{Fr}$ & $(-)$ & 0.25 & 0.25 \\
\hline Reynolds number & $\operatorname{Re}$ & $(-)$ & $1.215 \mathrm{E} 09$ & 9.824E06 \\
\hline
\end{tabular}

modification vector $\delta_{s}$ is used for both problems, since it is deemed more efficient in the context of shape design optimization [19].

Geometric constraints include fixed length between perpendicular and fixed displacement $(\nabla)$, with beam $(B)$ and $\operatorname{draft}(T)$ varying between $\pm 5 \%$ of the original hull. Fixed $\angle B P$ and $\nabla$ are satisfied by automatic geometric scaling, while constraints for $B$ and $T$ are handled using a penalty function method. This is used here, since the relationship between beam/draft variations and design variables is not explicitly provided by the orthogonal expansion and geometric scaling.

\subsection{Problem I}

\subsubsection{Design modification method}

The shape modification $\boldsymbol{\delta}_{s}$ is defined using $N=6$ orthogonal basis functions of the curvilinear coordinates $\xi$ and $\eta$ over the (demi) hull

$\boldsymbol{\psi}_{j}(\xi, \eta): \mathcal{S}=\left[0, L_{\xi}\right] \times\left[0, L_{\eta}\right] \in \mathbb{R}^{2} \longrightarrow \mathbb{R}^{3}, \quad j=1, \ldots, N$

as

$\boldsymbol{\delta}_{s}(\xi, \eta)=\sum_{j=1}^{N} \alpha_{j} \boldsymbol{\psi}_{j}(\xi, \eta)$ where the coefficients $\alpha_{j} \in \mathbb{R}(j=1, \ldots, N)$ are the design variables,

$\boldsymbol{\psi}_{j}(\xi, \eta):=\sin \left(\frac{r_{j} \pi \xi}{L_{\xi}}+\phi_{j}\right) \sin \left(\frac{t_{j} \pi \eta}{L_{\eta}}+\chi_{j}\right) \mathbf{e}_{q(j)}$

and the following orthogonality property is imposed:

$\iint_{\mathcal{S}} \boldsymbol{\psi}_{i}(\xi, \eta) \cdot \boldsymbol{\psi}_{j}(\xi, \eta) d \xi d \eta=\delta_{i j}$

In Eq. (11), $r_{j}$ and $t_{j} \in \mathbb{R}$ define the order of the function in $\xi$ and $\eta$ direction, respectively; $\phi_{j}$ and $\chi_{j} \in \mathbb{R}$ are the corresponding spatial phases; $L_{\xi}$ and $L_{\eta} \in \mathbb{R}$ define the domain size; $\mathbf{e}_{q(j)}$ is a unit vector. Modifications may be applied in $x, y$, or $z$ direction, with $q(j)=1$, 2 , or 3 , respectively. Four functions and design variables are used for the hull, whereas two functions/variables are used for the sonar dome, as summarized in Table 2 . The corresponding basis functions are shown in Fig. 3. Upper and lower bounds used for the design variables $\alpha_{j}$ are included in Table 2 .

\subsubsection{Hydrodynamic analysis}

Simulations are conducted using the code WARP (Wave Resistance Program), developed at CNR-INSEAN. Wave resistance computations are based on linear potential flow theory using Neumann-Kelvin linearization. Details of equations, numerical implementations, and validation of the numerical solver are given in [20]. The wave resistance is evaluated with the transverse wave cut method [21,22], whereas the frictional resistance is estimated using a flat-plate approximation, based on the local Reynolds number [23]. The steady 2DOF (sinkage and trim) equilibrium is achieved by iteration of the flow solver and the body equation of motion.

Simulations are performed for the right demi hull, taking advantage of symmetry about the $x z$-plane. The computational domain for the free-surface is shown in Fig. 4a. The associated panel grid used (Fig. 4b) is summarized in Table 3 and guarantees solution convergence. The validation of the potential flow analyses performed by WARP for the original hull is shown in Fig. 5 versus experimental (EFD) data collected at CNR-INSEAN [34], showing a reasonable agreement especially for low speeds; $C_{T}=R_{T} / 0.5 \rho U^{2} S_{w, s t a t}, \sigma / L B P$,

Table 2

Problem I, orthogonal function parameters for shape modification.

\begin{tabular}{|c|c|c|c|c|c|c|c|c|}
\hline Description & $j$ & $r_{j}$ & $\phi_{j}$ & $t_{j}$ & $\chi_{j}$ & $q(j)$ & $\alpha_{j, \min }(\mathrm{m})$ & $\alpha_{j, \max }(\mathrm{m})$ \\
\hline \multirow{4}{*}{ Hull modification } & 1 & 2.0 & 0 & 1.0 & 0 & 2 & -2.0 & 2.0 \\
\hline & 2 & 3.0 & 0 & 1.0 & 0 & 2 & -2.0 & 2.0 \\
\hline & 3 & 1.0 & 0 & 2.0 & 0 & 2 & -1.0 & 1.0 \\
\hline & 4 & 1.0 & 0 & 3.0 & 0 & 2 & -1.0 & 1.0 \\
\hline \multirow{2}{*}{ Sonar dome modification } & 5 & 1.0 & 0 & 1.0 & 0 & 2 & -0.6 & 0.6 \\
\hline & 6 & 0.5 & $\pi / 2$ & 0.5 & 0 & 3 & -1.0 & 1.0 \\
\hline
\end{tabular}
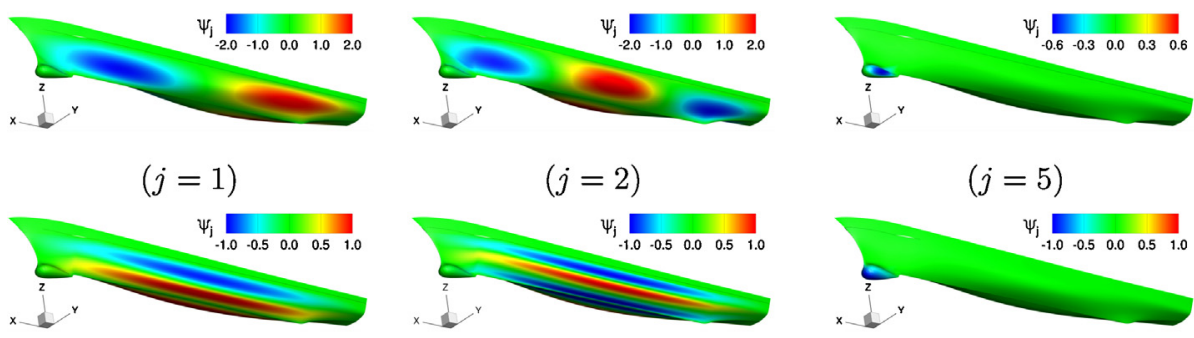

$(j=3)$

$(j=4)$

$(j=6)$

(a) Hull modification

(b) Sonar dome modification

Fig. 3. Problem I, orthogonal functions $\psi_{j}(\xi, \eta)$. 


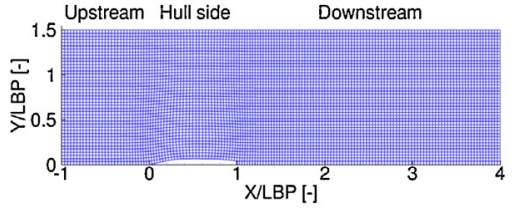

(a) Free-surface

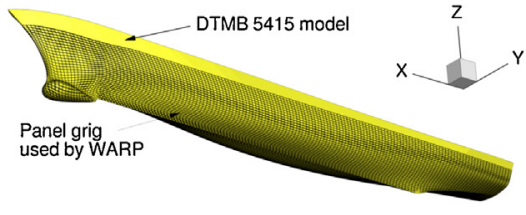

(b) Hull

Fig. 4. Computational panel grid for WARP.

(a)

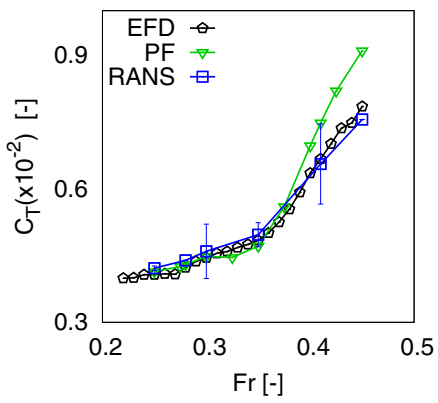

(b)

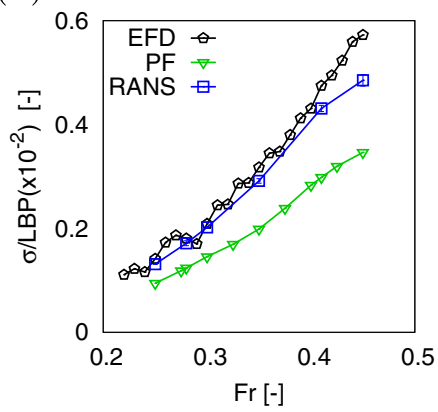

(c)

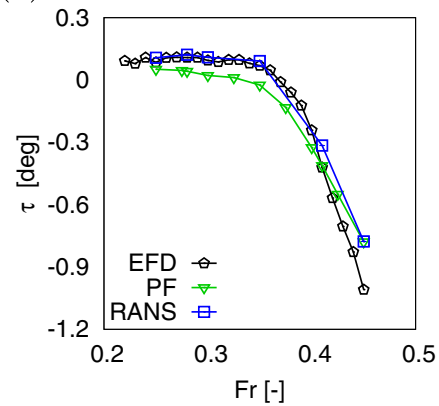

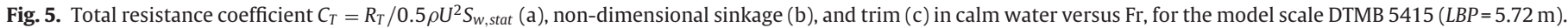

and $\tau$ are shown, where $U$ is the undisturbed flow speed, $S_{w, \text { stat }}$ is the static wetted surface area, $\sigma$ is the sinkage (positive if the center of gravity sinks), and $\tau$ is the trim angle (positive if the bow sinks).

For the hull form optimization process, a limit to the number of function evaluations is set equal to 1536 , i.e., $256 \mathrm{~N}$.

\subsection{Problem II}

\subsubsection{Design modification and dimensionality reduction methods}

The shape modification $\delta_{s}$ is defined using $M=27$ orthogonal basis functions of the Cartesian coordinates $x, y, z$ over a hyperrectangle

$\varphi_{j}(x, y, z): \mathcal{V}=\left[0, L_{x}\right] \times\left[0, L_{y}\right] \times\left[0, L_{z}\right] \in \mathbb{R}^{3} \longrightarrow \mathbb{R}^{3}, \quad j=1, \ldots, M$

as

$$
\boldsymbol{\delta}_{s}(x, y, z)=\sum_{j=1}^{M} \beta_{j} \varphi_{j}(x, y, z)
$$

where the coefficients $\beta_{j} \in \mathbb{R}(j=1, \ldots, M)$ are the design variables,

$\boldsymbol{\varphi}_{j}(x, y, z):=\sin \left(\frac{n_{j} \pi x}{L_{x}}+\phi_{j}\right) \sin \left(\frac{m_{j} \pi y}{L_{y}}+\chi_{j}\right) \sin \left(\frac{l_{j} \pi z}{L_{z}}+\theta_{j}\right) \mathbf{e}_{q(j)}$

and the following orthogonality property is imposed:

$\iiint_{\mathcal{V}} \varphi_{i}(x, y, z) \cdot \varphi_{j}(x, y, z) d x d y d z=\delta_{i j}$

Table 3

Computational panel grid for WARP.

\begin{tabular}{lllll}
\hline Hull & \multicolumn{2}{l}{ Free surface } & & \multirow{2}{*}{ Total } \\
\cline { 2 - 4 } & Upstream & Hull side & Downstream & \\
\hline $150 \times 30$ & $30 \times 44$ & $30 \times 44$ & $90 \times 44$ & $11 k$ \\
\hline
\end{tabular}

In Eq. (15), $n_{j}, m_{j}$, and $l_{j} \in \mathbb{R}$ define the order of the function in $x, y$, and $z$ direction, respectively; $\phi_{j}, \chi_{j}$, and $\theta_{j} \in \mathbb{R}$ are the corresponding spatial phases; $L_{x}, L_{y}$, and $L_{z} \in \mathbb{R}$ define the hyperrectangle; $\mathbf{e}_{q(j)}$ is a unit vector. Modifications may be applied in $x, y$, or $z$ direction, with $q(j)=1,2$, or 3 , respectively. Table 4 summarizes the parameters used herein.

The design space defined by Eq. (14) is reduced in dimensionality using the generalized KLE method presented in [25]:

$\boldsymbol{\delta}_{s}(\xi, \eta) \approx \sum_{j=1}^{N} \alpha_{j} \boldsymbol{\Phi}_{j}(\xi, \eta)$

Table 4

Problem II, orthogonal function parameters for shape modification.

\begin{tabular}{rlllllllll}
\hline$j$ & $n_{j}$ & $\phi_{j}$ & $m_{j}$ & $\chi_{j}$ & $l_{j}$ & $\theta_{j}$ & $q(j)$ & $\beta_{j, \min }(\mathrm{m})$ & $\beta_{j, \max }(\mathrm{m})$ \\
\hline 1 & 1.0 & 0 & 1.0 & 0 & 1.0 & 0 & 2 & -1.0 & 1.0 \\
2 & 1.0 & 0 & 1.0 & 0 & 2.0 & 0 & 2 & -1.0 & 1.0 \\
3 & 1.0 & 0 & 2.0 & 0 & 1.0 & 0 & 2 & -1.0 & 1.0 \\
4 & 2.0 & 0 & 1.0 & 0 & 1.0 & 0 & 2 & -1.0 & 1.0 \\
5 & 1.0 & 0 & 2.0 & 0 & 2.0 & 0 & 2 & -1.0 & 1.0 \\
6 & 2.0 & 0 & 1.0 & 0 & 2.0 & 0 & 2 & -1.0 & 1.0 \\
7 & 2.0 & 0 & 2.0 & 0 & 1.0 & 0 & 2 & -1.0 & 1.0 \\
8 & 2.0 & 0 & 2.0 & 0 & 2.0 & 0 & 2 & -1.0 & 1.0 \\
9 & 1.0 & 0 & 1.0 & 0 & 3.0 & 0 & 2 & -1.0 & 1.0 \\
10 & 1.0 & 0 & 3.0 & 0 & 1.0 & 0 & 2 & -1.0 & 1.0 \\
11 & 3.0 & 0 & 1.0 & 0 & 1.0 & 0 & 2 & -1.0 & 1.0 \\
12 & 1.0 & 0 & 2.0 & 0 & 3.0 & 0 & 2 & -1.0 & 1.0 \\
13 & 2.0 & 0 & 1.0 & 0 & 3.0 & 0 & 2 & -1.0 & 1.0 \\
14 & 1.0 & 0 & 3.0 & 0 & 2.0 & 0 & 2 & -1.0 & 1.0 \\
15 & 2.0 & 0 & 3.0 & 0 & 1.0 & 0 & 2 & -1.0 & 1.0 \\
16 & 3.0 & 0 & 1.0 & 0 & 2.0 & 0 & 2 & -1.0 & 1.0 \\
17 & 3.0 & 0 & 2.0 & 0 & 1.0 & 0 & 2 & -1.0 & 1.0 \\
18 & 2.0 & 0 & 2.0 & 0 & 3.0 & 0 & 2 & -1.0 & 1.0 \\
19 & 2.0 & 0 & 3.0 & 0 & 2.0 & 0 & 2 & -1.0 & 1.0 \\
20 & 3.0 & 0 & 2.0 & 0 & 2.0 & 0 & 2 & -1.0 & 1.0 \\
21 & 1.0 & 0 & 3.0 & 0 & 3.0 & 0 & 2 & -1.0 & 1.0 \\
22 & 3.0 & 0 & 1.0 & 0 & 3.0 & 0 & 2 & -1.0 & 1.0 \\
23 & 3.0 & 0 & 3.0 & 0 & 1.0 & 0 & 2 & -1.0 & 1.0 \\
24 & 2.0 & 0 & 3.0 & 0 & 3.0 & 0 & 2 & -1.0 & 1.0 \\
25 & 3.0 & 0 & 2.0 & 0 & 3.0 & 0 & 2 & -1.0 & 1.0 \\
26 & 3.0 & 0 & 3.0 & 0 & 2.0 & 0 & 2 & -1.0 & 1.0 \\
27 & 3.0 & 0 & 3.0 & 0 & 3.0 & 0 & 2 & -1.0 & 1.0 \\
\hline & & & & & & & & &
\end{tabular}



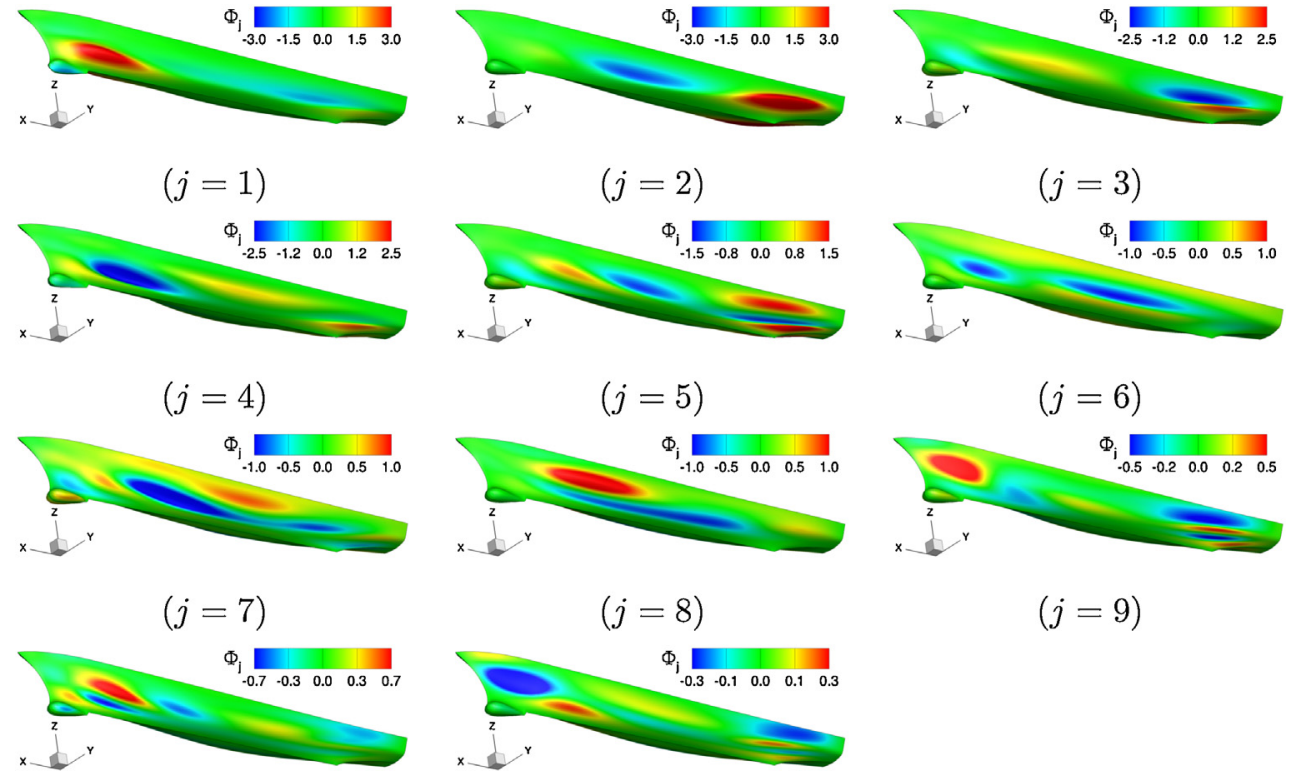

$(j=9)$

$(j=10)$

$(j=11)$

Fig. 6. Problem II, KLE solutions $\Phi_{j}(x, y, z)$.

where $\xi$ and $\eta$ are curvilinear coordinates over the (demi) hull, $\alpha_{j} \in$ $\mathbb{R}(j=1, \ldots, N)$ are new design variables and $\boldsymbol{\Phi}_{j}$ are the solutions of the eigenproblem

$\iint_{\mathcal{S}} \rho\left(\xi^{\prime}, \eta^{\prime}\right)\left\langle\boldsymbol{\delta}_{S}(\xi, \eta) \otimes \boldsymbol{\delta}_{s}\left(\xi^{\prime}, \eta^{\prime}\right)\right\rangle$

$\boldsymbol{\Phi}_{j}\left(\xi, \eta^{\prime}\right) d \xi^{\prime} d \eta^{\prime}=\lambda_{j} \boldsymbol{\Phi}_{j}(\xi, \eta)$

provided that $\left\langle\boldsymbol{\delta}_{s}(\xi, \eta, \boldsymbol{\alpha})\right\rangle=\mathbf{0}, \forall(x, y, z) \in \mathcal{V}$. The brackets $\langle\cdot\rangle$ indicate ensemble average over the realizations of the design variable vector $\boldsymbol{\beta}=\left\{\beta_{j}\right\} \in \mathcal{L}$, which is assessed here using 10,000 uniform random samples (each of them resulting in a modified hull form). The weight $\rho \in \mathbb{R}^{+}$defines a generalized inner product and is used to give more emphasis to submerged grid nodes. The following orthogonality property holds [35,36]

$\iint_{\mathcal{S}} \rho(\xi, \eta) \boldsymbol{\Phi}_{i}(\xi, \eta) \cdot \boldsymbol{\Phi}_{j}(\xi, \eta) d x d y d z=\delta_{i j}$

Finally, the reduced dimension $N$ is selected in order to retain the $90 \%$ of the original geometric variance, as

$\sum_{j=1}^{N} \lambda_{j} \geq 0.90 \sum_{k=1}^{\infty} \lambda_{k}$

provided that $\lambda_{1} \geq \lambda_{2} \geq \cdots \geq \lambda_{j} \geq \lambda_{j+1}$. For the current problem, $N=11$ and the corresponding eigenfunctions (represented on the hull) are shown in Fig. 6. Details of the formulation and numerical implementation of the design space dimensionality reduction technique may be found in [25]. Details of the application to the hull form optimization of the DTMB 5415 may be found in [26].

\subsubsection{Hydrodynamic analysis and metamodelling}

RANS simulations are performed with the CFDShip-Iowa v4.5 code [24], which has the capability of a 6DOF simulation and has been developed at the University of Iowa, IIHR-Hydroscience \& Engineering, over the past 25 years. The SST blended $k-\epsilon / k-\omega$ turbulent model is used. The free-surface location is predicted by a single phase level set method. A second order upwind scheme is used to discretize the convective terms of momentum equations.
For a high performance parallel computing, an MPI-based domain decomposition approach is used, where each decomposed block is mapped to one processor. The code SUGGAR runs as a separate process from the flow solver to compute interpolation coefficients for the overset grid, which enables CFDShip-Iowa to take care of $6 \mathrm{DOF}$ with a motion controller at every time step. Only 2DOFs are considered in the current study.

Simulations are performed for the right demi hull, taking advantage of symmetry about the $x z$-plane. Table 5 summarizes the associated background and boundary layer volume grids used, designed to have $y^{+}=0.3$ at $\mathrm{Fr}=0.25$. The computational domain and grids are shown in Figs. 7 and 8. The validation of the RANS analyses performed by CFDShip-Iowa for the original hull is shown in Fig. 5 versus the EFD data collected at CNR-INSEAN [34], showing a good agreement. It may be noted how the agreement is particularly good at low speeds, for which the domain and grid were designed. Error bars indicate the grid uncertainty, evaluated using the factor of safety method [37].

The optimization problem is solved using a metamodel, trained by 71 RANS simulations defined using a sequential sampling procedure [10]. The metamodel used is a first order polyharmonic spline, which is a special case of radial basis function (RBF) interpolation [9].

For the hull form optimization process, a limit to the number of metamodel-based function evaluations is set equal to 8800 , i.e., $800 N$.

\subsubsection{Automatic boundary layer grid modification}

The boundary layer (volume) grid is automatically modified, in order to reflect the shape modification applied to the body (surface) grid. Assume that the body surface grid is defined with index $J=1$ and spanned by indices $I=1, \ldots, I_{\max }$ and $K=1, \ldots, K_{\max }$. Accordingly, the boundary layer grid is spanned by $I=1, \ldots, I_{\max }, J=1, \ldots$,

Table 5

Computational volume grid for CFDShip-Iowa.

\begin{tabular}{lll}
\hline Background & Boundary layer & Total \\
\hline $227 \times 155 \times 115$ & $243 \times 71 \times 115$ & $6 M$ \\
\hline
\end{tabular}




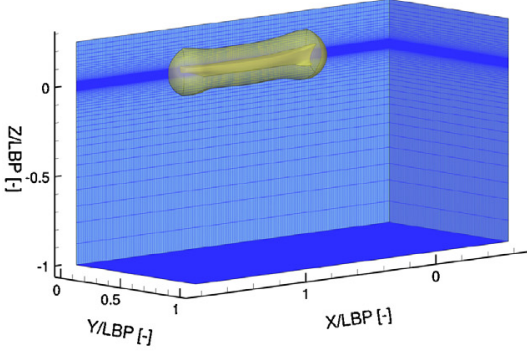

(a) Background

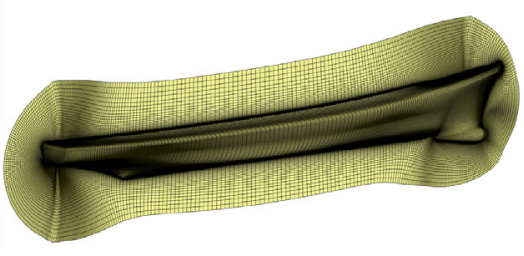

(b) Boundary layer

Fig. 7. Computational volume grid for CFDShip-Iowa.

$J_{\max }$, and $K=1, \ldots, K_{\max }$, with $J=J_{\max }$ corresponding to the outer surface. Once the grid nodes of the body surface at $J=1$ are modified as per Eq. (17), any arbitrary inner node of the boundary layer grid $\left(J=2, \ldots, J_{\max }-1\right)$ is modified similarly to Eq. (8), as

$\mathbf{x}=\mathbf{x}_{0}+\boldsymbol{\delta}$

with

$\delta=\frac{l^{*}-l}{l^{*}} \boldsymbol{\delta}_{s}+\frac{l}{l^{*}} \boldsymbol{\delta}_{s}^{*}$

where $l$ is the distance between (original) inner and body surface nodes, with arbitrary $J$ and $J=1$, respectively (and same $I$ and $K$ indices); $l^{*}$ is the distance between (original) outer and body surface nodes, with $J=J_{\max }$ and $J=1$, respectively (and same $I$ and $K$ indices); $\delta_{s}^{*}$ is the modification of the outer surface $\left(J=J_{\max }\right)$ :

$\delta_{s}^{*}=c \delta_{S}$

with $c \in \mathbb{R}_{0}^{+}$.
The distance $l$ (and $l^{*}$ ) may be evaluated in the simplest form as the Euclidean distance $\bar{l}$ :

$l=\bar{l}=\left\|\mathbf{x}_{0}-\mathbf{x}_{s, 0}\right\|$

Alternatively, the approximate curvilinear distance $\hat{l}$ along the grid line at constant $I$ and $K$ may be used [38]:

$l=\hat{l}=\sum_{j=1}^{J-1}\left\|\mathbf{x}_{0}^{(j+1)}-\mathbf{x}_{0}^{(j)}\right\|$

where superscripts indicate grid indices, limited to $J$ for the sake of compactness (since $I$ and $K$ are constant).

In the current work, $c=0$ in Eq. (23) and $l=\bar{l}$ in Eq. (22). During the metamodel training, each modified grid is assessed for quality by means of $y^{+}$, determinant of the Jacobian matrix and skewness.

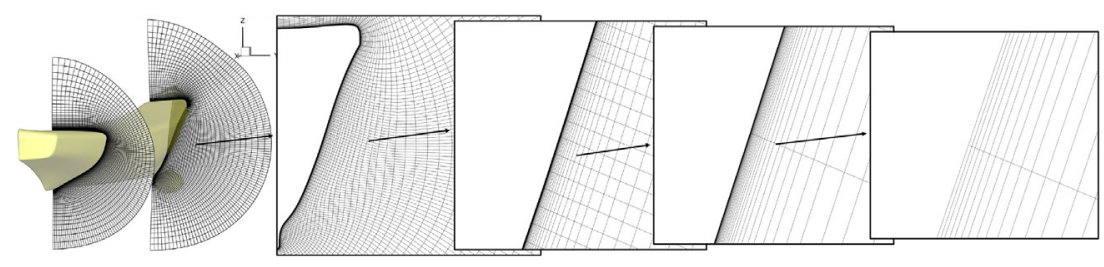

(a) Original hull

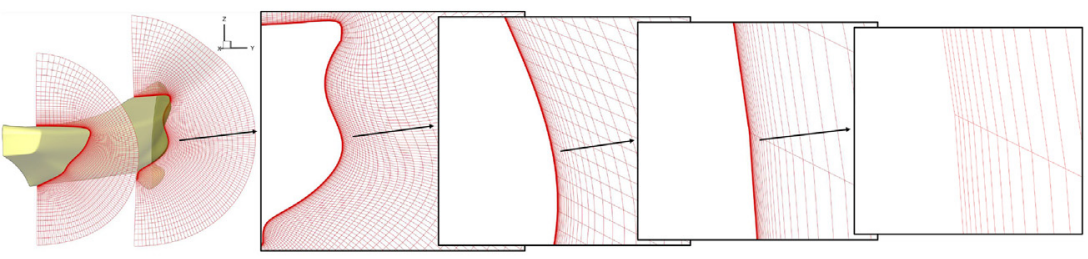

(b) Modified hull laying on the design space boundary $\left(x_{1}=1\right)$

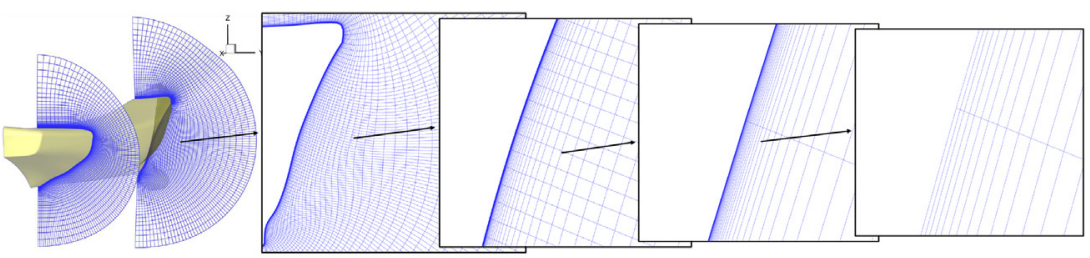

(c) Optimized hull

Fig. 8. Close view of the boundary layer grid (sections at constant $I$ ). 

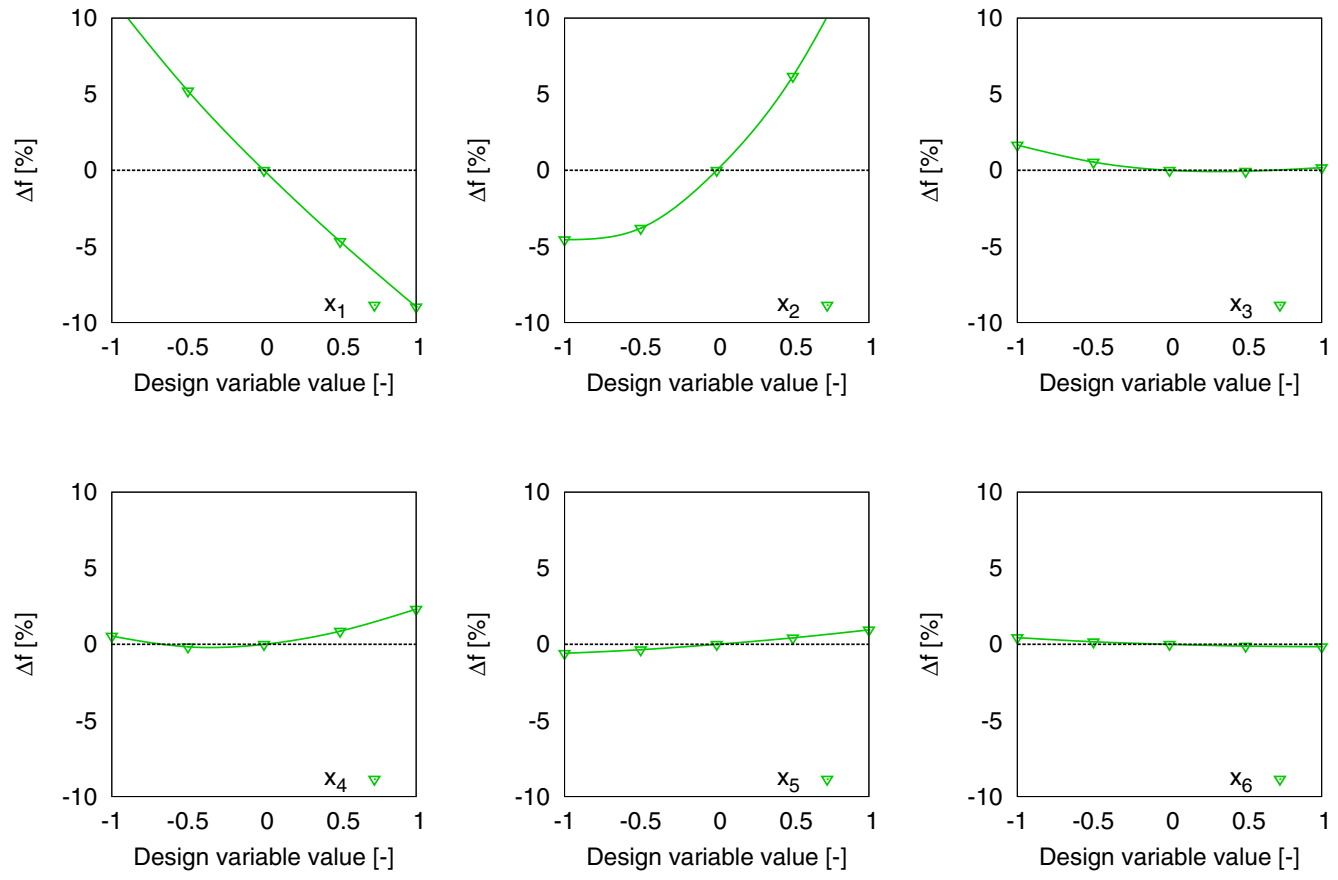

Fig. 9. Problem I, sensitivity analysis of non-dimensional design variables.

\section{Numerical results}

\subsection{Problem I}

A preliminary sensitivity analysis for each design variable is presented in Fig. 9, showing the associated percent resistance reduction $(\Delta f)$ with respect to the original hull. Non-dimensional design variables are shown in the plots, $x_{j}=2\left(\alpha_{j}-\ell_{j}\right) /\left(u_{j}-\ell_{j}\right)-1$. Changes in $f$ are found significant for all variables but $x_{5}$ and $x_{6}$, revealing a possible reduction of the total resistance at $\mathrm{Fr}=0.25$ close to $10 \%$. The analysis of the results is conducted setting apart results (i) for a low budget of 192 function evaluations (which corresponds to $32 \mathrm{~N}$, an eighth of the full budget), and (ii) for the full budget of 1536 function evaluations (which corresponds to $256 \mathrm{~N}$ ).

For the case (i), the optimization procedure achieves a resistance reduction of $13.7 \%$ and $15.5 \%$ using DIRECT and DIRMIN-2, respectively, and a reduction of $13.5 \%$ and $16.0 \%$ using DPSO and LS-DF_PSO, respectively. The two global/local hybrid algorithms outperform their global version. In particular, LS-DF_PSO is found the most efficient algorithm for the present SBD problem, achieving the best design with the fastest convergence rate, as shown in Fig. 10. Fig. 11 presents the values of the optimized design
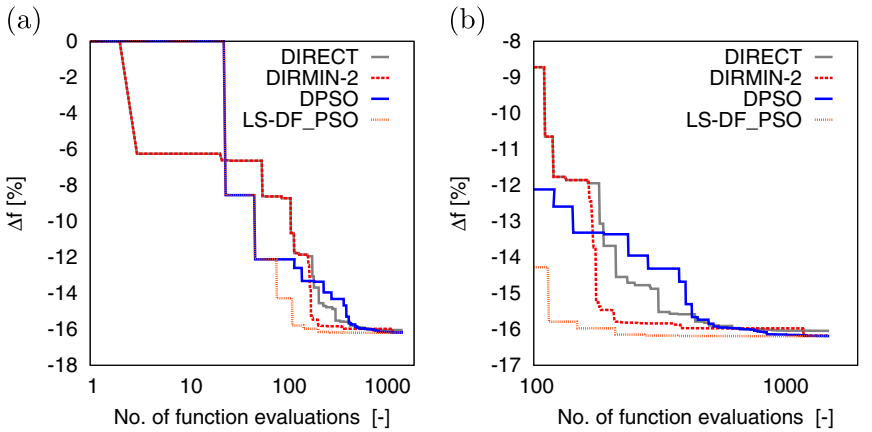

Fig. 10. Problem I, objective function convergence history (a) and detail after the first 100 function evaluations (b).

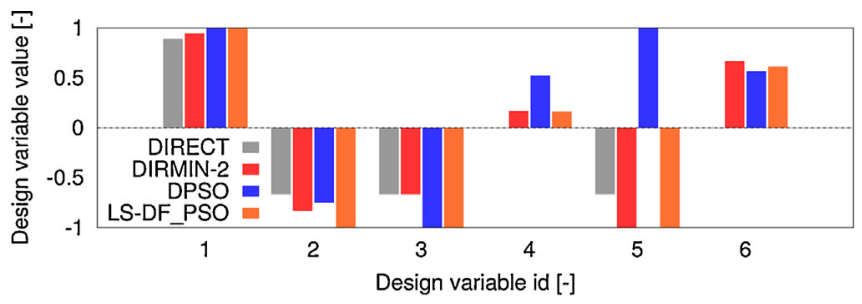

(a) Objective function convergence of optimized design variables

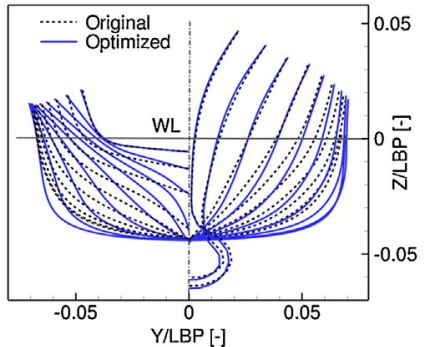

(b) DIRECT

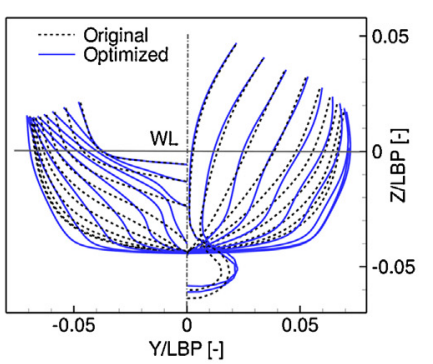

(d) DPSO

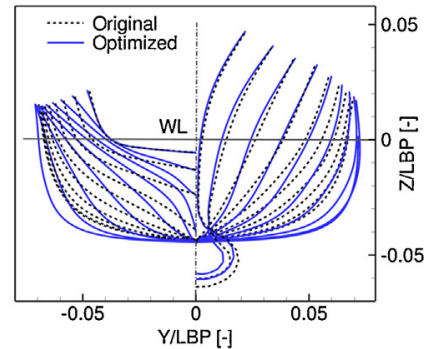

(c) DIRMIN-2

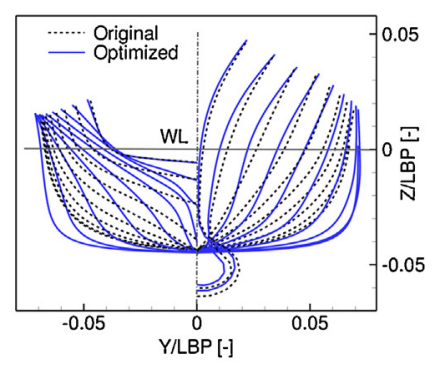

(e) LS-DF_PSO
Fig. 11. Problem I, 192 function evaluations. 
Table 6

Problem I, summary of the optimization results.

\begin{tabular}{|c|c|c|c|c|c|c|c|c|c|}
\hline \multirow[t]{2}{*}{ No. funct. evaluations } & \multirow[t]{2}{*}{ Algorithm } & \multicolumn{6}{|c|}{ Design variables (non-dimensional) } & \multicolumn{2}{|c|}{$R_{T}\left(\times 10^{5}\right)(\mathrm{N})$} \\
\hline & & $x_{1}$ & $x_{2}$ & $x_{3}$ & $x_{4}$ & $x_{5}$ & $x_{6}$ & Value & $\Delta f \%$ \\
\hline \multirow{4}{*}{192} & DIRECT & 0.89 & -0.67 & -0.67 & 0.00 & -0.67 & 0.00 & 2.96 & -13.7 \\
\hline & DIRMIN-2 & 0.94 & -0.83 & -0.67 & 0.17 & -1.00 & 0.67 & 2.92 & -15.5 \\
\hline & DPSO & 1.00 & -0.75 & -1.00 & 0.52 & 1.00 & 0.56 & 2.96 & -13.5 \\
\hline & LS-DF_PSO & 1.00 & -1.00 & -1.00 & 0.16 & -1.00 & 0.61 & 2.89 & -16.0 \\
\hline \multirow{4}{*}{1536} & DIRECT & 1.00 & -0.92 & -0.67 & 0.11 & -1.00 & 0.31 & 2.88 & -16.0 \\
\hline & DIRMIN-2 & 1.00 & -0.94 & -0.77 & 0.17 & -1.00 & 0.67 & 2.88 & -16.2 \\
\hline & DPSO & 1.00 & -0.99 & -0.97 & 0.29 & -1.00 & 0.69 & 2.88 & -16.2 \\
\hline & LS-DF_PSO & 1.00 & -1.00 & -0.86 & 0.16 & -0.99 & 0.75 & 2.88 & -16.2 \\
\hline
\end{tabular}

variables, showing appreciable differences, and shows the corresponding optimized shapes, compared to the original.

For the case (ii), the optimization procedure achieves a resistance reduction of $16.0 \%$ and $16.2 \%$ using DIRECT and DIRMIN-2, respectively, and a reduction of $16.2 \%$ using both DPSO and LSDF_PSO. The convergence history of the objective function towards the minimum is shown in Fig. 10, confirming the efficiency and robustness of the two hybrid global/local approaches DIRMIN-2 and LS-DF_PSO. More in detail, LS-DF_PSO achieves the most significant reduction of the objective function overall, although all the solutions are very close in this case. Fig. 12 presents the values of the corresponding optimized design variables and shows the optimized shapes compared to the original. The close agreement of the solutions obtained by the different algorithms indicates that the global minimum region has been likely achieved. A summary of the optimization results is presented in Table 6.

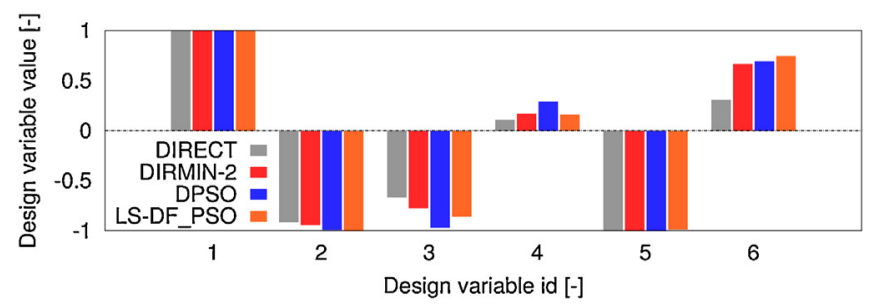

(a) Objective function convergence of optimized design variables

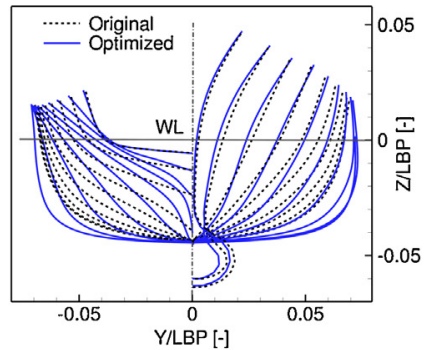

(b) DIRECT

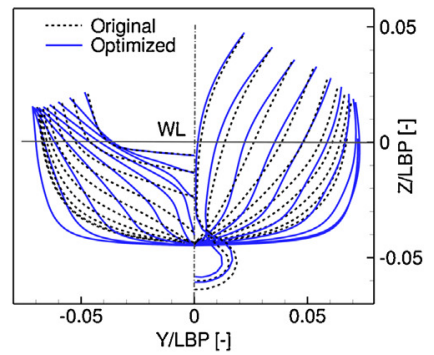

(d) DPSO

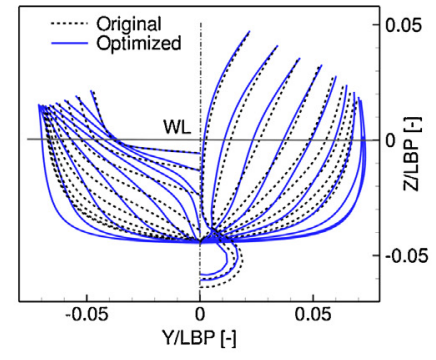

(c) DIRMIN-2

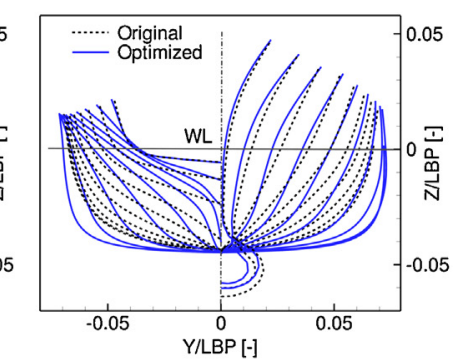

(e) LS-DF_PSO
Fig. 12. Problem I, 1536 function evaluations.

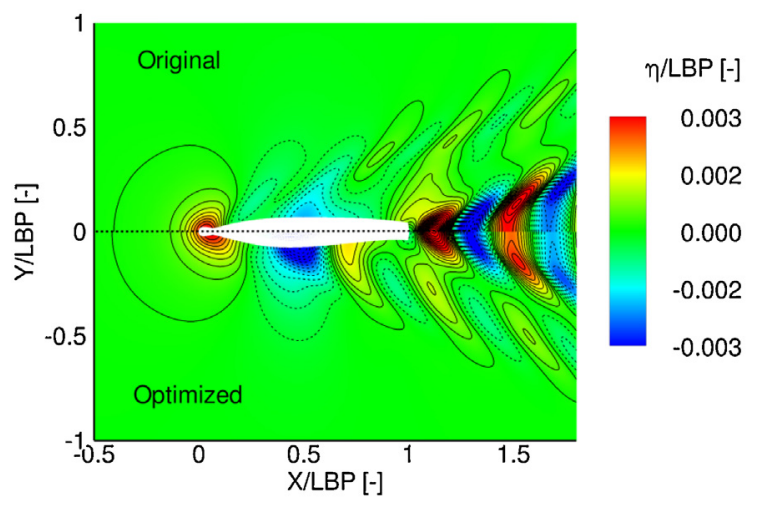

Fig. 13. Problem I, wave patterns produced by optimized hull forms at $\mathrm{Fr}=0.25$ compared with original.

The reduction of the wave elevation pattern of the final shape, both in terms of transverse and diverging stern waves, is visible in Fig. 13. Finally, Fig. 14 presents the pressure field on the optimized hulls compared to the original hull, showing a better pressure recovery towards the stern. Obviously, the solver is not able to predict flow separations, which are likely to occur for such large design modifications.

\subsection{Problem II}

A preliminary sensitivity analysis for each design variable is presented in Fig. 15, showing the associated percent resistance reduction $(\Delta f)$ with respect to the original hull. The quality of the grids produced by the method presented in Section 3.2.3 is assessed for each modified design. Fig. $8 \mathrm{~b}$ presents a modified grid laying on the boundary of the design space, showing an acceptable quality.

Non-dimensional design variables are shown in the plots, $x_{j}=2\left(\alpha_{j}-\ell_{j}\right) /\left(u_{j}-\ell_{j}\right)-1$. Changes in $f$ are found significant for all variables, revealing a possible reduction of the total resistance at $\mathrm{Fr}=0.25$ close to $5 \%$. Variations are smaller than Problem I, due to more realistic simulations by the RANS solver. The analysis of the results is conducted setting apart results (i) for a low budget of 1100 function evaluations (which corresponds to $100 \mathrm{~N}$, an eighth of the

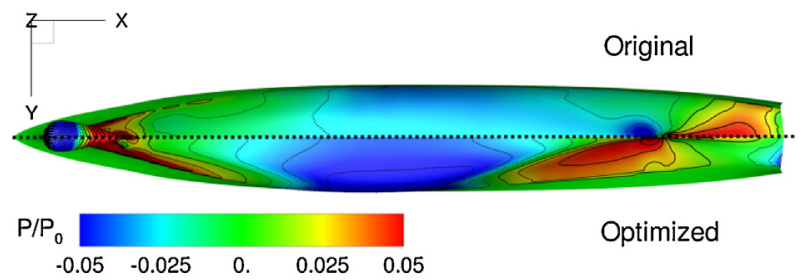

Fig. 14. Problem I, pressure field on optimized hull forms at $\mathrm{Fr}=0.25$ compared with original. 

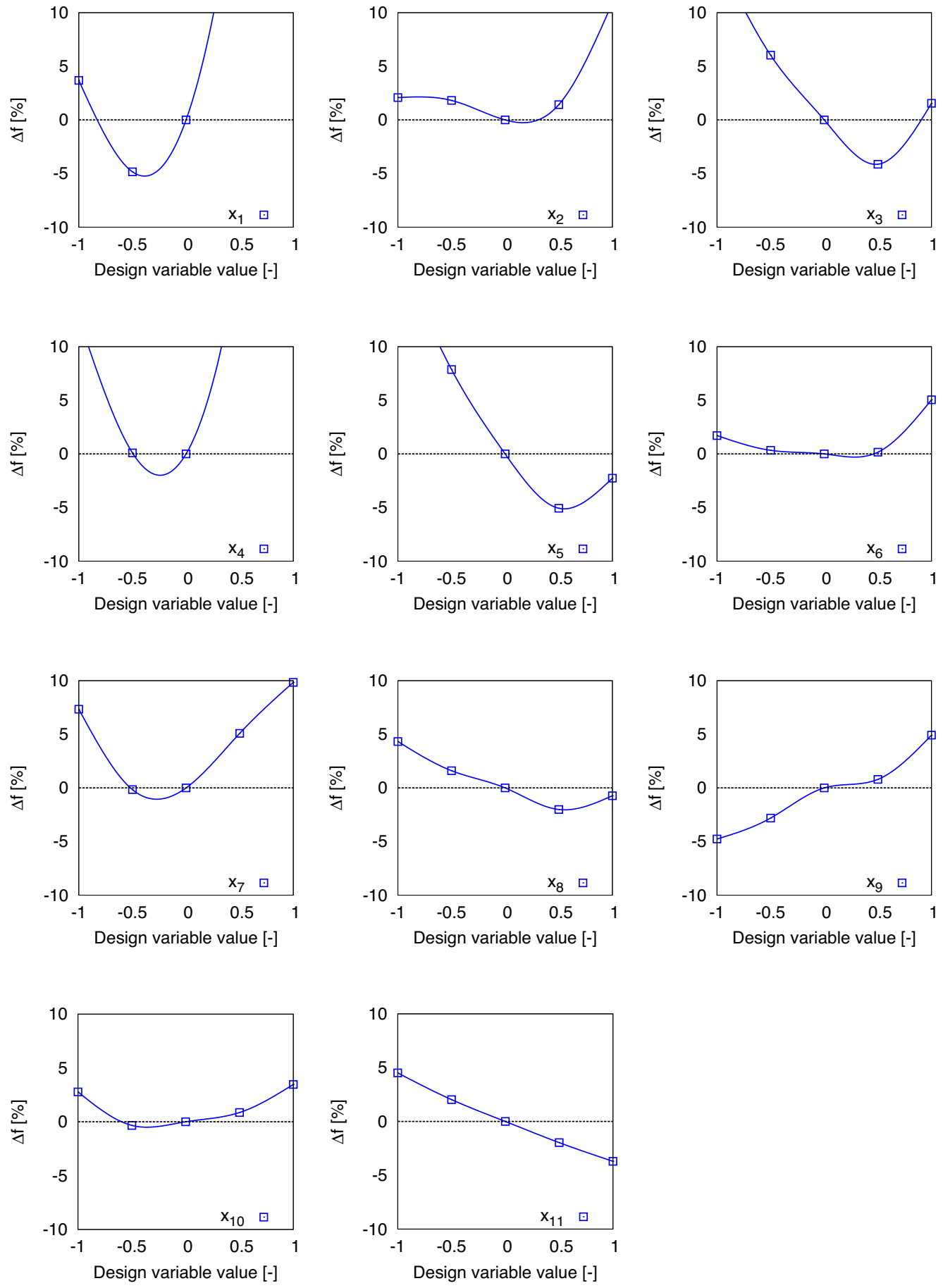

Fig. 15. Problem II, sensitivity analysis of non-dimensional design variables.

full budget), and (ii) for the full budget of 8800 function evaluations (which corresponds to $800 \mathrm{~N}$ ).

For the case (i), the optimization procedure achieves a total resistance coefficient reduction of $5.16 \%$ and $5.37 \%$ using DIRECT and DIRMIN-2, respectively, and a reduction of $4.98 \%$ using both DPSO and LS-DF_PSO. DIRMIN-2 outperform its global version, whereas DPSO and its hybrid reach the same result because non a local search has been activated by LS-DF_PSO. DIRMIN-2 is found the most efficient algorithm for the present SBD problem, achieving the best design with the fastest convergence rate, as shown in Fig. 16. Fig. 17 presents the values of the optimized design variables, showing appreciable differences, and shows the corresponding optimized shapes, compared to the original.
For the case (ii), the optimization procedure achieves a resistance reduction of 5.95\% and 5.98\% using DIRECT and DIRMIN-2, respectively, and a reduction of $5.52 \%$ and $5.91 \%$ using DPSO and LS-DF_PSO, respectively. The convergence history of the objective function towards the minimum is shown in Fig. 16, confirming the efficiency and robustness of the two hybrid global/local approaches DIRMIN-2 and LS-DF_PSO. More in detail, DIRMIN-2 achieves the most significant reduction of the objective function overall, although all the solutions are very close in this case. Fig. 18 presents the values of the corresponding optimized design variables and shows the optimized shapes compared to the original. The close agreement of the solutions obtained by the different algorithms indicates that the global minimum region has been likely 
Table 7

Problem II, summary of the optimization results.

\begin{tabular}{|c|c|c|c|c|c|c|c|c|c|c|c|c|c|c|}
\hline \multirow[t]{2}{*}{ No. funct. evaluations } & \multirow[t]{2}{*}{ Algorithm } & \multicolumn{11}{|c|}{ Design variables (non-dimensional) } & \multicolumn{2}{|c|}{$C_{T}\left(\times 10^{-3}\right)(-)$} \\
\hline & & $x_{1}$ & $x_{2}$ & $x_{3}$ & $x_{4}$ & $x_{5}$ & $x_{6}$ & $x_{7}$ & $x_{8}$ & $x_{9}$ & $x_{10}$ & $x_{11}$ & Value & $\Delta f \%$ \\
\hline \multirow{4}{*}{1100} & DIRECT & -0.22 & -0.07 & 0.00 & -0.07 & 0.44 & 0.07 & 0.00 & 0.15 & 0.07 & -0.07 & 0.07 & 4.00 & -5.16 \\
\hline & DIRMIN-2 & -0.28 & -0.03 & 0.03 & -0.16 & 0.12 & -0.03 & -0.03 & 0.13 & 0.17 & -0.09 & 0.40 & 3.99 & -5.37 \\
\hline & DPSO & -0.30 & 0.11 & 0.09 & -0.14 & 0.11 & -0.05 & -0.04 & -0.10 & -0.31 & -0.08 & -0.05 & 4.01 & -4.98 \\
\hline & LS-DF_PSO & -0.30 & 0.11 & 0.09 & -0.14 & 0.11 & -0.05 & -0.04 & -0.10 & -0.31 & -0.08 & -0.05 & 4.01 & -4.98 \\
\hline \multirow{4}{*}{8800} & DIRECT & -0.15 & -0.01 & 0.01 & -0.11 & 0.05 & -0.03 & -0.01 & 0.02 & -0.77 & -0.03 & 0.01 & 3.97 & -5.95 \\
\hline & DIRMIN-2 & -0.15 & -0.01 & 0.01 & -0.10 & 0.05 & -0.03 & -0.02 & 0.02 & -0.77 & -0.03 & 0.02 & 3.97 & -5.98 \\
\hline & DPSO & -0.21 & -0.02 & 0.04 & -0.11 & 0.06 & -0.05 & -0.04 & 0.12 & -0.61 & -0.07 & -0.03 & 3.99 & -5.52 \\
\hline & LS-DF_PSO & -0.15 & -0.06 & 0.02 & -0.09 & 0.06 & -0.03 & -0.03 & 0.03 & -0.77 & -0.04 & -0.01 & 3.97 & -5.91 \\
\hline
\end{tabular}

(a)

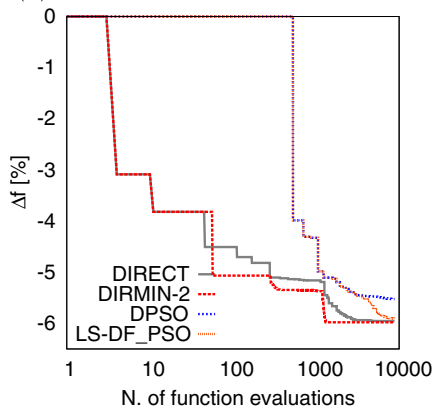

(b)

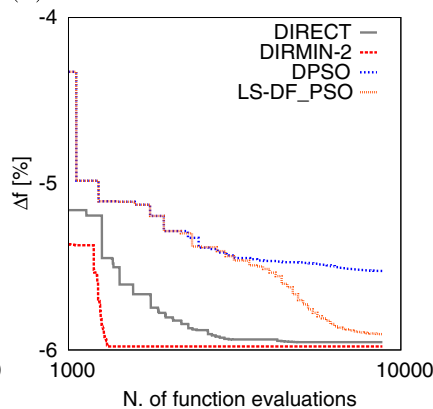

Fig. 16. Problem II, objective function convergence history (a) and detail after the first 1000 function evaluations (b).

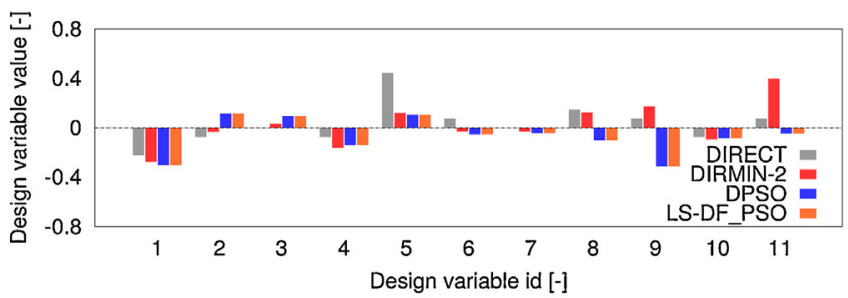

(a) Objective function convergence of optimized design variables

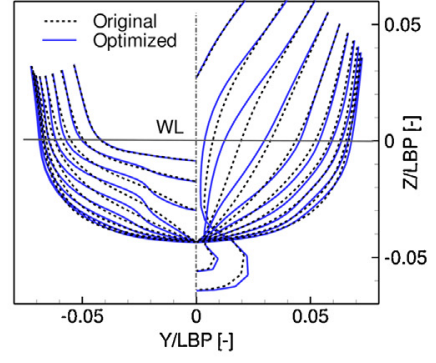

(b) DIRECT

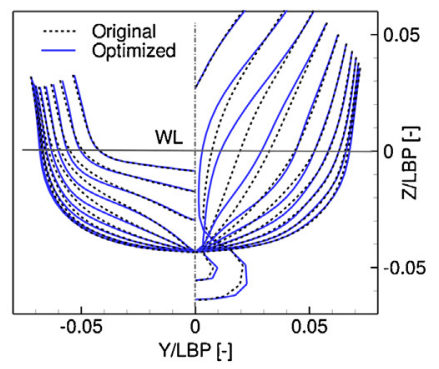

(d) DPSO

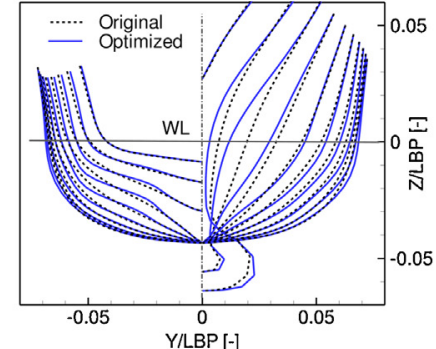

(c) DIRMIN-2

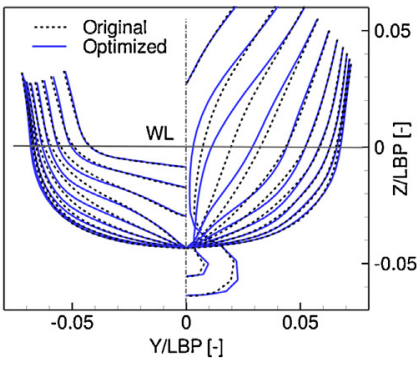

(e) LS-DF_PSO
Fig. 17. Problem II, 1100 function evaluations. achieved. A summary of the optimization results is presented in Table 7.

The best design is finally assessed with RANS. The associated modified grid is assessed and presented in Fig. 8c, showing a good quality. The results are presented in Figs. 19-21, and 22, and Table 8. Figs. 19 and 20 show a significant reduction of the diverging bow wave and a small reduction of the diverging and transverse stern wave. It may be also noted how the shoulder wave is cancelled. Specifically, the optimized shoulder shape induces a high pressure region in correspondence of the first trough of the original hull, causing a phase shift with the reduction of the diverging bow wave and the cancellation of the shoulder wave (well visible in Fig. 20). This effect has been also shown in retrofitting studies by optimization of blisters attached to the original hull [39]. A longitudinal wave cut along the $y=0.1 \mathrm{LBP}$ plane is shown in Fig. 21, highlighting the reduction of the wave elevation, especially at the bow.

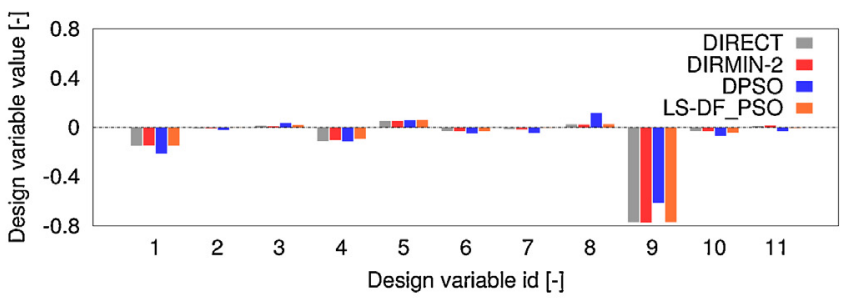

(a) Objective function convergence of optimized design variables

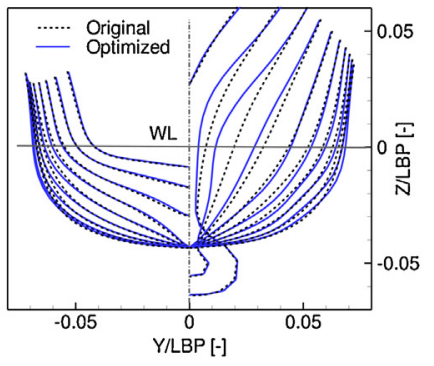

(b) DIRECT

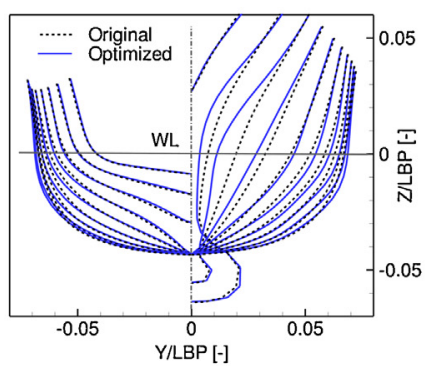

(d) DPSO

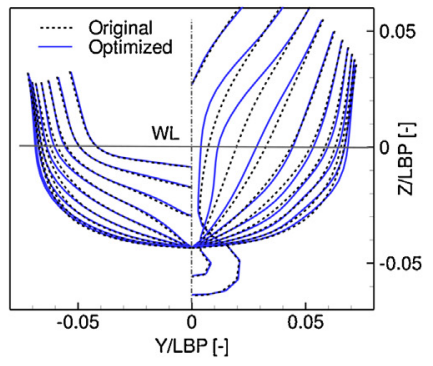

(c) DIRMIN-2

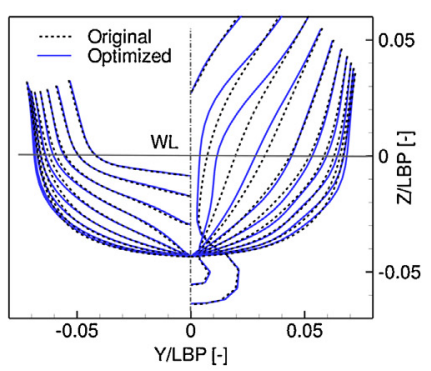

(e) LS-DF_PSO
Fig. 18. Problem II, 8800 function evaluations. 
Table 8

Problem II, comparison between original and optimized DTMB 5415 hydrodynamic coefficients $\left(C_{p p}\right.$ represent the piezometric pressure, $C_{h}$ the hydrostatic pressure, $C_{f}$ the viscous shear stress, $C_{m g, x}$ the component of the weight force along the longitudinal axis, and $C_{T}$ the total resistance).

\begin{tabular}{llccc}
\hline Parameter & Unit & \multicolumn{1}{c}{ Original } & Optimized & \multicolumn{1}{c}{$\Delta \%$} \\
\hline$C_{p p}$ & $(-)$ & $1.38 \mathrm{E}-03$ & $9.08 \mathrm{E}-04$ & -34.0 \\
$C_{h}$ & $(-)$ & $0.86 \mathrm{E}-03$ & $1.24 \mathrm{E}-03$ & 42.0 \\
$C_{f}$ & $(-)$ & $3.16 \mathrm{E}-03$ & $3.18 \mathrm{E}-03$ & 0.65 \\
$C_{m g, x}$ & $(-)$ & $-1.19 \mathrm{E}-03$ & $-1.35 \mathrm{E}-03$ & -13.4 \\
$C_{T}$ & $(-)$ & $4.21 \mathrm{E}-03$ & $3.97 \mathrm{E}-03$ & -6.00 \\
$\sigma / L B P$ & $(-)$ & $-1.31 \mathrm{E}-03$ & $-1.35 \mathrm{E}-03$ & -3.29 \\
$\tau$ & $(\mathrm{deg})$ & -0.11 & -0.12 & -15.3 \\
$S_{w, \text { stat }} / L \mathrm{LP}^{2}$ & $(-)$ & $1.48 \mathrm{E}-02$ & $1.50 \mathrm{E}-02$ & 0.96 \\
\hline
\end{tabular}

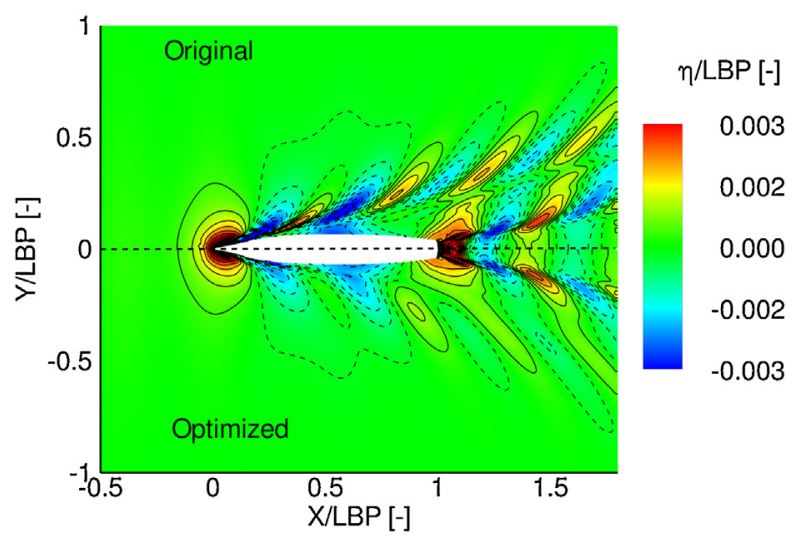

Fig. 19. Problem II, wave patterns produced by optimized hull forms at $\mathrm{Fr}=0.25$ compared with original.

Finally, Fig. 22 presents the pressure field on the optimized shape compared to the original shape, showing a more uniform pressure distribution along the hull and a slightly better pressure recovery at the stern. The hydrodynamic coefficients for the original and the

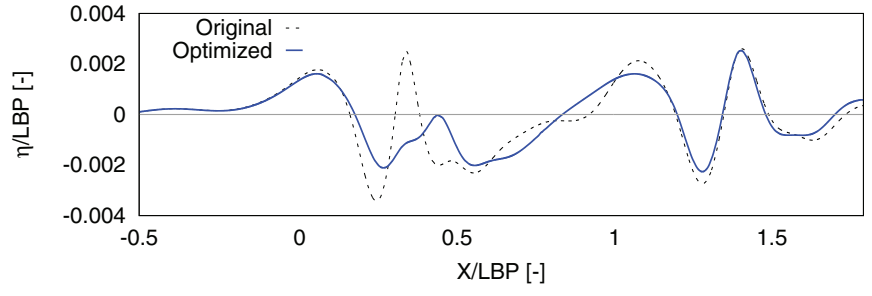

Fig. 21. Problem II, longitudinal wave cut on the $y=0.1 \mathrm{LBP}$ plane at $\mathrm{Fr}=0.25$ for optimized and original hulls.

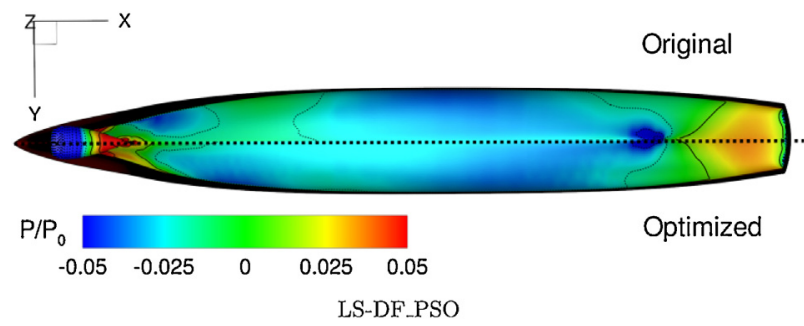

Fig. 22. Problem II, pressure field on optimized hull forms at $\mathrm{Fr}=0.25$ compared with original.

optimized hulls are finally compared in Table 8, confirming that a large part of the resistance reduction stems from the reduction of the piezometric pressure coefficient.

\section{Conclusions}

A deterministic derivative-free global design optimization of the DTMB 5415 hull form has been shown, using global/local hybridization by derivative-free line search methods of two well-known global algorithms, DIRECT and DPSO, respectively.

The optimization has been performed aiming at the reduction of the total resistance (consequently the total resistance coefficient)

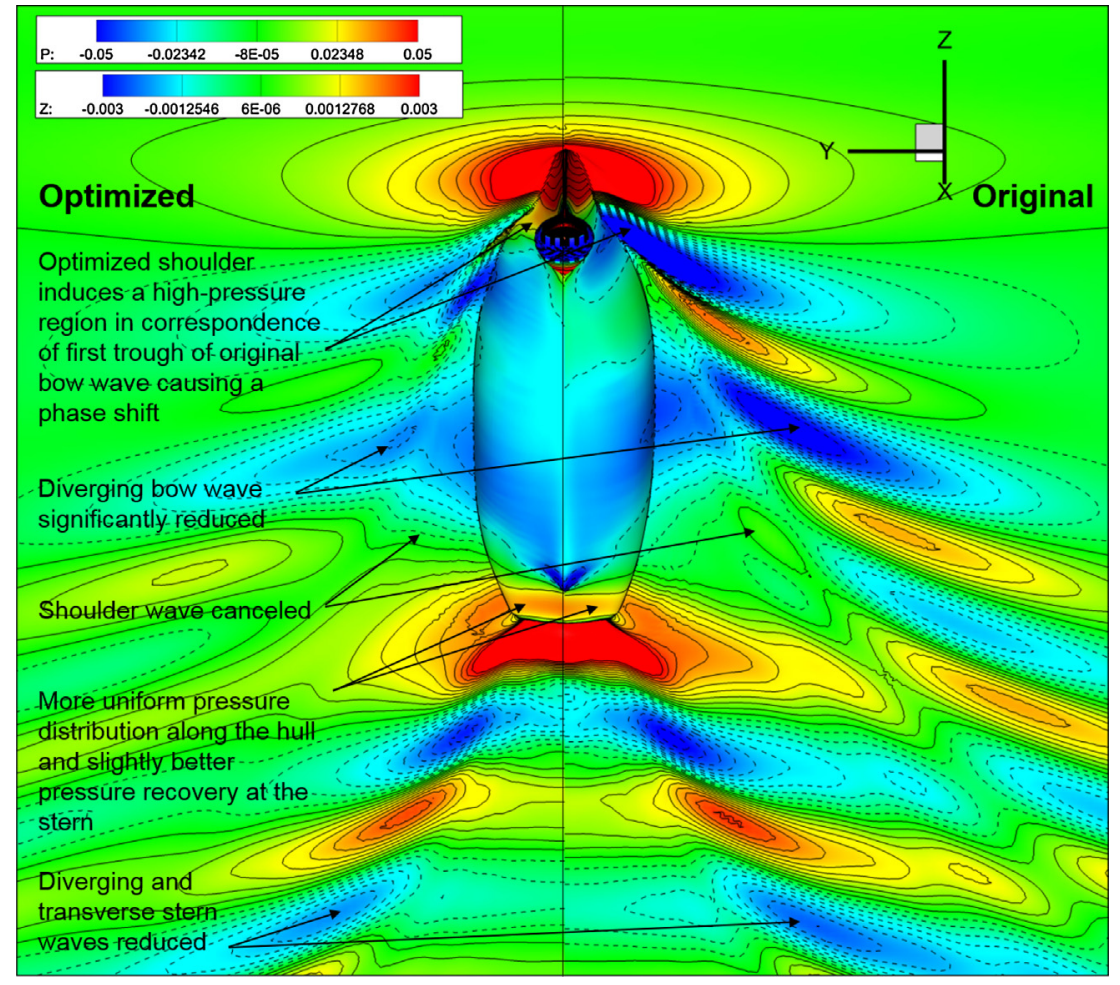

Fig. 20. Problem II, bottom view of wave pattern and pressure distribution at $\mathrm{Fr}=0.25$ for optimized (left) and original (right) hulls. 
in calm water at $\mathrm{Fr}=0.25$, using two design spaces generated by an expansion of orthogonal basis functions for the modification of the hull form. Two problems (I and II) have been solved with a number of design variables equal to six and eleven, respectively. Computer simulations were conducted using a linear potential flow code and a RANS solver (problem I and II, respectively).

A resistance reduction of $16 \%$ and $6 \%$ has been achieved by the optimized designs for problem I and II. As obvious, the potential flow solver is not able to assess the design performance when significant flow separations occur, as likely happening in some region of the current design space. The final shape provided by the potential flow appears therefore quite unrealistic. Furthermore, compared to RANS, the current potential flow implementation is not able to predict accurately the bow wave, which is found under predicted. Nevertheless, the pressure distribution on the original hull is reasonable and the validation versus experimental data for the original hull is also acceptable.

The RANS-based optimization relies on more accurate highfidelity hydrodynamic analyses and provides a more realistic hull form. The final shape obtained with RANS induces a high pressure region in correspondence of the first trough of the diverging bow wave of the original hull. This causes a phase shift with a significant reduction of the bow wave and the cancellation of the shoulder wave. As a result, the pressure distribution appears more uniformly distributed along the hull and most of the resistance reduction stems from the piezometric pressure coefficient.

For problem I (with six design variables), the hybrid methods reach solutions close to convergence using about 200 function evaluations, while the original algorithms require almost twice the number of evaluations. For problem II (with 11 design variables), the hybrid methods reach convergence using about 1100 function calls, whereas the original algorithms seem to need more than 10,000 evaluations to reach convergence. The increased number of evaluations needed by problem II versus I arises from spanning a higher dimensional design space and (as in the present study) suggests the use of metamodels to reduce the computational cost. Nevertheless, latest studies [40] have shown the solution of a stochastic multi-objective optimization of a high-speed catamaran, using a number of 780 RANS simulations in calm water and 1170 in regular waves, with a grid size of 6.9M. This demonstrates the technological possibility of applying directly the optimization algorithm to RANS simulations, even if the number of function calls becomes larger than 1000 , as for current problem II. In this case, the efficiency of the optimization algorithm is a crucial issue and (in view of current results) hybrid methods may represent a viable and valid option.

In conclusion, the present research has shown how global/local hybridization methods, namely DIRMIN-2 and LS-DF_PSO, outperform their original global algorithms, DIRECT and DPSO. This result has been found significant especially for low budgets of function evaluations. Hybrid algorithms have shown their capability to combine effectively the characteristics of global and local approaches, resulting in a faster (and computationally less expensive) convergence towards the global minimum. This, along with their derivative-free formulation and implementation, makes the present local hybridization methods a viable and effective option for SBD optimization, especially when computationally expensive objective functions are involved. The final hydrodynamic assessment of the RANS based optimized shape has confirmed the effectiveness of the SBD optimization procedure, driven by hybrid global/local methods.

Future work will focus on the stochastic optimization of the DTMB 5415 hull form, subject to real ocean environment and operations. In this context, the extension of hybrid global/local methods to multi-objective problems will be addressed and combined with metamodel-based optimization by adaptive sampling procedures.

\section{Acknowledgments}

The work has been performed in collaboration with NATO STO Task Groups AVT-204 "Assess the Ability to Optimize Hull Forms of Sea Vehicles for Best Performance in a Sea Environment" and AVT252 "Stochastic Design Optimization for Naval and Aero Military Vehicles." The authors are grateful to Dr. Woei-Min Lin and Dr. KiHan Kim of the US Navy Office of Naval Research, for their support through NICOP grant N62909-15-1-2016 and grant N00014-14-10195. The authors are also grateful to the Italian Flagship Project RITMARE, coordinated by the Italian National Research Council and founded by the Italian Ministry of Education. The authors would like to thank Dr. Shanti Bhushan for providing the computational grid for RANS simulations of the original DTMB 5415 model. Finally, the authors would like to thank the anonymous reviewers for their valuable comments and suggestions to improve the quality of the paper.

\section{References}

[1] E.F. Campana, D. Peri, Y. Tahara, F. Stern, Shape optimization in ship hydrodynamics using computational fluid dynamics, Comput. Methods Appl. Mech. Eng. 196 (1-3) (2006) 634-651

[2] S. Percival, D. Hendrix, F. Noblesse, Hydrodynamic optimization of ship hull forms, Appl. Ocean Res. 23 (6) (2001) 337-355.

[3] M.A. Martins, E.N. Lages, E.S. Silveira, Compliant vertical access riser assessment: DOE analysis and dynamic response optimization, Appl. Ocean Res. 41 (2013) 28-40.

[4] I. Noad, R. Porter, Optimisation of arrays of flap-type oscillating wave surge converters, Appl. Ocean Res. 50 (2015) 237-253

[5] E.F. Campana, G. Liuzzi, S. Lucidi, D. Peri, V. Piccialli, A. Pinto, New global optimization methods for ship design problems, Optim. Eng. 10 (4) (2009) 533-555.

[6] R. Shafaghat, S. Hosseinalipour, I. Lashgari, A. Vahedgermi, Shape optimization of axisymmetric cavitators in supercavitating flows, using the NSGA II algorithm, Appl. Ocean Res. 33 (3) (2011) 193-198.

[7] M.P. Aghababa, 3D path planning for underwater vehicles using five evolutionary optimization algorithms avoiding static and energetic obstacles, Appl. Ocean Res. 38 (2012) 48-62.

[8] E. Uzlu, M. İhsan Kömürcü, M. Kankal, T. Dede, H.T. Öztürk, Prediction of berm geometry using a set of laboratory tests combined with teaching-learning-based optimization and artificial bee colony algorithms, Appl. Ocean Res. 48 (2014) 103-113.

[9] S. Volpi, M. Diez, N. Gaul, H. Song, U. Iemma, K.K. Choi, E.F. Campana, F. Stern, Development and validation of a dynamic metamodel based on stochastic radial basis functions and uncertainty quantification, Struct. Multidiscip. Optim. 51 (2) (2015) 347-368.

[10] M. Diez, S. Volpi, A. Serani, F. Stern, E.F. Campana, Simulation-based design optimization by sequential multi-criterion adaptive sampling and dynamic radial basis functions, in: EUROGEN 2015, International Conference on Evolutionary and Deterministic Methods for Design, Optimization and Control with Applications to Industrial and Societal Problems, Glasgow, UK, September, 2015

[11] E.F. Campana, G. Fasano, A. Pinto, Dynamic analysis for the selection of parameters and initial population, in particle swarm optimization, J. Glob. Optim. 48 (3) (2010) 347-397.

[12] D. Jones, C. Perttunen, B. Stuckman, Lipschitzian optimization without the Lipschitz constant, J. Optim. Theory Appl. 79 (1) (1993) 157-181.

[13] A. Serani, M. Diez, C. Leotardi, D. Peri, G. Fasano, U. Iemma, E.F. Campana, On the use of synchronous and asynchronous single-objective deterministic particle swarm optimization in ship design problems, in: Proceedings of the 1st International Conference in Engineering and Applied Sciences Optimization, Kos, Greece, June 4-6, 2014.

[14] E.F. Campana, M. Diez, U. Iemma, G. Liuzzi, S. Lucidi, F. Rinaldi, A. Serani, Derivative-free global ship design optimization using global/local hybridization of the DIRECT algorithm, Optim. Eng. 17 (1) (2015) $127-156$

[15] A. Serani, M. Diez, E.F. Campana, G. Fasano, D. Peri, U. Iemma, Globally convergent hybridization of particle swarm optimization using line search-based derivative-free techniques, in: X.-S. Yang (Ed.), Recent Advances in Swarm Intelligence and Evolutionary Computation, Studies in Computational Intelligence, vol. 585, Springer International Publishing, 2015, pp. 25-47.

[16] F. Stern, J. Longo, R. Penna, A. Olivieri, T. Ratcliffe, H. Coleman, International collaboration on benchmark CFD validation data for surface combatant DTMB model 5415, in: Proceedings of the Twenty-Third Symposium on Naval Hydrodynamics, Val de Reuil, France, September 17-22, 2000.

[17] J. Longo, F. Stern, Uncertainty assessment for towing tank tests with example for surface combatant DTMB model 5415, J. Ship Res. 49 (1) (2005) 55-68. 
[18] Y. Tahara, D. Peri, E.F. Campana, F. Stern, Computational fluid dynamics-based multiobjective optimization of a surface combatant using a global optimization method, J. Mar. Sci. Technol. 13 (2008) 95-116.

[19] M. Diez, A. Serani, E.F. Campana, O. Goren, K. Sarioz, D.B. Danisman, G. Grigoropoulos, E. Aloniati, M. Visonneau, P. Queutey, F. Stern, Multi-objective hydrodynamic optimization of the DTMB 5415 for resistance and seakeeping, in: Proceedings of the 13th International Conference on Fast Sea Transportation, FAST 2015, Washington, DC, USA, 2015.

[20] P. Bassanini, U. Bulgarelli, E.F. Campana, F. Lalli, The wave resistance problem in a boundary integral formulation, Surv. Math. Ind. 4 (1994) 151-194

[21] K.W.H. Eggers, S.D. Sharma, L.W. Ward, An assessment of some experimental methods for the determination of the wavemaking characteristics of a ship form, Tech. Rep. 184, Institut für Schiffbau der Uniuversität Hamburg, 1967.

[22] J. Telste, A. Reed, Calculation of transom stern flows, in: Proceedings of the Sixth International Conference on Numerical Ship Hydrodynamics, 1994, pp. $78-92$.

[23] H. Schlichting, K. Gersten, Boundary-Layer Theory, Springer-Verlag, Berlin, 2000.

[24] J. Huang, P.M. Carrica, F. Stern, Semi-coupled air/water immersed boundary approach for curvilinear dynamic overset grids with application to ship hydrodynamics, Int. J. Numer. Methods Fluids 58 (6) (2008) 591-624.

[25] M. Diez, E.F. Campana, F. Stern, Design-space dimensionality reduction in shape optimization by Karhunen-Loève expansion, Comput. Methods Appl. Mech. Eng. 283 (2015) 1525-1544.

[26] M. Diez, A. Serani, E.F. Campana, F. Stern, Design space dimensionality reduction for single- and multi-disciplinary shape optimization, in: AIAA/ISSMO Multidisciplinary Analysis and Optimization (MA\&O), AVIATION 2016, Washington, DC, USA, June 13-17, 2016.

[27] A. Serani, G. Fasano, G. Liuzzi, S. Lucidi, U. Iemma, E.F. Campana, M. Diez, Derivative-free global design optimization in ship hydrodynamics by local hybridization, in: Proceedings of the 14th International Conference on Computer Applications and Information Technology in the Maritime Industries, Ulrichshusen, Germany, May 11-13, 2015, pp. 331-342.

[28] S. Lucidi, M. Sciandrone, A derivative-free algorithm for bound constrained optimization, Comput. Optim. Appl. 21 (2) (2002) 119-142.
[29] J. Kennedy, R. Eberhart, Particle swarm optimization, in: Proceedings of the Fourth IEEE Conference on Neural Networks, Piscataway, NJ, 1995, pp. 1942-1948.

[30] A. Serani, C. Leotardi, U. Iemma, E. Campana, G. Fasano, M. Diez, Parameter selection in synchronous and asynchronous deterministic particle swarm optimization for ship hydrodynamics problems, Appl. Soft Comput. (2016), submitted for publication.

[31] T.T. Wong, W.S. Luk, P.A. Heng, Sampling with Hammersley and Halton points, J. Graphics Tools 2 (2) (1997) 9-24.

[32] M. Clerc, Stagnation analysis in particle swarm optimization or what happens when nothing happens, Tech. rep., 2006 http://hal.archives-ouvertes.fr/hal00122031.

[33] I. Trelea, The particle swarm optimization algorithm: convergence analysis and parameter selection, Inf. Process. Lett. 85 (2003) 317-325.

[34] A. Olivieri, F. Pistani, A. Avanzini, F. Stern, R. Penna, Towing tank, sinkage and trim, boundary layer, wake, and free surface flow around a naval combatant INSEAN 2340 model, Tech. rep., DTIC, 2001.

[35] U. Iemma, L. Morino, M. Diez, Digital holography and Karhunen-Loève decomposition for the modal analysis of two-dimensional vibrating structures, J. Sound Vib. 291 (1) (2006) 107-131.

[36] U. Iemma, M. Diez, L. Morino, An extended Karhunen-Loève decomposition for modal identification of inhomogeneous structures, J. Vib. Acoust. 128 (2006) 357.

[37] T. Xing, F. Stern, Factors of safety for Richardson extrapolation, J. Fluids Eng. $132(6)(2010) 061403$

[38] K.-J. Paik, P.M. Carrica, D. Lee, K. Maki, Strongly coupled fluid-structure interaction method for structural loads on surface ships, Ocean Eng. 36 (17-18) (2009) 1346-1357.

[39] M. Kandasamy, P. Wu, S. Zalek, D. Karr, S. Bartlett, L. Nguyen, F. Stern, CFD based hydrodynamic optimization and structural analysis of the hybrid ship hull, in: SNAME Trans., 2014.

[40] M. Diez, E.F. Campana, F. Stern, Development and evaluation of hull-form stochastic optimization methods for resistance and operability, in: Proceedings of the 13th International Conference on Fast Sea Transportation, FAST 2015, Washington, DC, USA, 2015. 